\title{
Loads Correlation of a Full-Scale UH-60A Airloads Rotor in a Wind Tunnel
}

\author{
Hyeonsoo Yeo \\ Ethan A. Romander \\ Aeroflightdynamics Directorate (AMRDEC) \\ U.S. Army Research, Development, and Engineering Command \\ Ames Research Center, Moffett Field, California \\ Flight Vehicle Research and Technology Division \\ NASA Ames Research Center \\ Moffett Field, California
}

\begin{abstract}
Wind tunnel measurements of the rotor trim, blade airloads, and structural loads of a full-scale UH-60A Black Hawk main rotor are compared with calculations obtained using the comprehensive rotorcraft analysis CAMRAD II and a coupled CAMRAD II/OVERFLOW 2 analysis. A speed sweep at constant lift up to an advance ratio of 0.4 and a thrust sweep at constant speed into deep stall are investigated. The coupled analysis shows significant improvement over comprehensive analysis. Normal force phase is better captured and pitching moment magnitudes are better predicted including the magnitude and phase of the two stall events in the fourth quadrant at the deeply stalled condition. Structural loads are, in general, improved with the coupled analysis, but the magnitude of chord bending moment is still significantly underpredicted. As there are three modes around 4 and 5/rev frequencies, the structural responses to the 5/rev airloads due to dynamic stall are magnified and thus care must be taken in the analysis of the deeply stalled condition.
\end{abstract}

\section{Nomenclature}

$C_{L} \quad$ rotor lift coefficient

$C_{T} \quad$ rotor thrust coefficient

$M^{2} C_{N} \quad$ nondimensional section normal force

$M^{2} C_{M} \quad$ nondimensional section pitching moment

$\alpha_{c} \quad$ wall-effect corrected shaft angle

$\alpha_{s} \quad$ geometric shaft angle (positive for rearward tilt)

$\beta_{o} \quad$ coning, deg

$\beta_{1 c} \quad$ longitudinal flapping, deg

$\beta_{1 s} \quad$ lateral flapping, deg

$\mu \quad$ advance ratio

$\sigma \quad$ solidity, 0.0826

$\theta_{o} \quad$ collective, deg

$\theta_{1 c} \quad$ lateral cyclic, deg

$\theta_{1 s} \quad$ longitudinal cyclic, deg

\section{Introduction}

A full-scale wind tunnel test of a UH-60A rotor was recently conducted (May 2010) by NASA and the U.S. Army in the National Full-Scale Aerodynamics Complex (NFAC) 40- by 80-Foot Wind Tunnel to provide an expanded database for validating and enhancing rotorcraft aeromechanics prediction methodology [1]. This test acquired a comprehensive set

Presented at the American Helicopter Society 68th Annual Forum, Fort Worth, Texas, May 1 - 3, 2012. This is a work of the U.S. Government and is not subject to copyright protection in the U.S. of validation-quality measurements on a full-scale pressureinstrumented rotor system at conditions that challenge the most sophisticated modeling and simulation tools. Figure 1 shows these UH-60A rotor blades installed on the NFAC Large Rotor Test Apparatus (LRTA) in the wind tunnel test section.

Data were acquired over a wide range of operating conditions, including conditions of airspeed up to $175 \mathrm{kt}$, thrust up to $32,000 \mathrm{lb}$, and advance ratio up to 1.0 . The database provides aerodynamic pressures, structural loads, control positions, and rotor forces and moments, allowing for the validation of both aerodynamic and structural models. These unique and extensive test data sets provide a useful resource that can be used to examine the rotor behavior in the most challenging conditions.

For the present investigation, two parametric sweeps were selected for analysis. First, a speed sweep was selected to test simulation accuracy over a wide range of advance ratios. Second, a thrust sweep was selected to test simulation accuracy under conditions extending from unstalled through some deep stall conditions. The rotor blade aerodynamic environment at high speed is characterized by compressibility and a poor distribution of loading over the rotor disk. At high thrust, aerodynamics are dominated by dynamic stall and large negative pitching moment.

Romander et al. [2] provided an initial correlation of airloads with measured data for the same test sets considered in the present paper using the coupled CAMRAD II [3] and OVERFLOW 2 [4]. In the present paper, both CAMRAD II 
alone and coupled CAMRAD II and OVERFLOW 2 analyses are carried out and rotor structural loads as well as airloads results are compared with the UH-60A rotor wind tunnel test data. Detailed examination and parametric studies are also conducted for the deeply stalled condition, where the previous analysis [2] had difficulty getting good correlation.

\section{Description of the Test}

The experiment was conducted in the NFAC 40- by 80 -Foot Wind Tunnel using a UH-60A rotor system mounted on the NFAC LRTA. The blades used in this test program were the same 4 rotor blades flown during the UH-60A Airloads Program [5]. A detailed description of the experiment can be found in Ref. 1, including information on the test hardware, instrumentation, data acquisition and reduction systems, rotor control systems, and standard test procedures. Information relevant to the current study is provided below.

\section{Instrumentation}

Two of these blades were heavily instrumented: one with 242 pressure transducers and one with a mix of strain-gages and accelerometers. The pressure blade was originally built with 242 sub-miniature pipette type absolute pressure transducers embedded below the skin surface of the blade. Absolute pressures were measured at nine radial locations $(\mathrm{r} / \mathrm{R}=0.225$, $0.40,0.55,0.675,0.775,0.865,0.92,0.965$, and 0.99) as shown in Fig. 2(a). Blade section normal force, pitching moment, and chord force were obtained by integrating the absolute pressures. For the blade airloads presented in this paper, only working transducers were included in the integrations. Detailed examination of pressures at each transducer are still in progress to ensure the accuracy of the integrated airloads.

Blade flap bending, chord bending, and torsion moments were measured with two- or four-leg strain-gage bridges bonded to the second instrumented blade. The gages were located at the blade root ( $11.3 \%$ radius) and then evenly distributed along the blade at $10 \%$ increments of the rotor radius $(20 \%$ to 90\%) as shown in Fig. 2(b). Most of the gauges were bonded directly to the blade spar and used during the Airloads flight test. Five additional torsion gages were bonded to the blade skin, including one at the same radial station as a spar-bonded gage to ensure measurement consistency.

The blade root motions occur around elastomeric bearings and the "hinges" are the focal points of the bearings. Two independent, specially designed blade motion measurement systems were used to determine blade flap, lag, and pitch angles at the root of each blade. The first system, designated the Blade Motion Hardware (BMH) or "crab arm", was used during the Airloads flight test. The BMH are mechanical measurement devices that span the main rotor blade hinge points and include three Rotary Variable
Differential Transformers (RVDTs) on each blade to measure the relative motions of its own articulations. The true blade motions are obtained through three kinematic equations that account for the cross-coupling between measurements. The second system, designated the Rotor State Measurement System, is composed of four sets of three laser distance transducers (one set mounted to each hub arm). Each transducer produces a laser beam that is reflected back to a sensor in the same enclosure by reflective objects within a specified distance range. The transducer then produces a voltage proportional to the distance to the object (and related to one of the blade root angles). The accuracy of these blade root motion measurement systems is currently under review.

\section{Test procedure}

A level flight speed sweep was conducted to provide data that showed the effects of advance ratio for representative flight conditions. For each wind tunnel test condition, rotor collective, cyclic, and shaft angle were adjusted to match the target lift, propulsive force, and hub moments. The 1-g level flight speed sweeps were performed at three lift levels, $C_{L} / \sigma=0.08,0.09$, and 0.10 , up to an advance ratio of 0.4 .

A thrust sweep was performed using specified rotor thrust and zero hub pitch and roll moments as trim targets until stall was detected. Then, collective pitch was manually varied to better define the phenomena. During the collective sweeps only zero hub moments were achieved (thrust not trimmed). The thrust sweeps were conducted at three different tip Mach numbers; $M_{t i p}=0.625,0.650$, and 0.675 . The majority of the data were acquired at the baseline $M_{t i p}=0.650$ with various shaft angles and advance ratios. A limited number of sweeps at $M_{t i p}=0.625$ were conducted to attain higher non-dimensional thrusts and advance ratios without reaching load limits.

In this paper, the speed sweep ranged from $\mu=0.15$ to 0.4 with a constant $C_{L} / \sigma=0.09$ and a tip Mach number of 0.65 (Run 52). The selected thrust sweep was at $\mu=0.3$ with a tip Mach number of 0.625 (Run 45). The test points are plotted in Fig. 3 along with aerodynamic rotor lift boundary obtained in wind tunnel testing of a model rotor by McHugh [6]. The maximum thrust obtained in this test is slightly higher than the McHugh lift boundary. Among those sweeps, five data points (circled in Fig. 3) were further selected for detailed analy sis. From the speed sweep, three points were selected; $\mu=0.2$ (Run 52, Point 20), $\mu=0.3$ (Run 52, Point 31), and $\mu=0.4$ (Run 52, Point 52) at $C_{L} / \sigma=0.09$. From the collective sweep, two points including the extreme thrust condition were selected; $C_{T} / \sigma=0.06$ (Run 45, Point 30) and $C_{T} / \sigma=0.1255$ (Run 45, Point 38) at $\mu=0.3$. 


\section{Description of Analytical Method}

The analytical results were obtained using the comprehensive analysis CAMRAD II alone and separately using coupled CAMRAD II/OVERFLOW 2. This section describes each method and how they are coupled to produce a higher fidelity solution.

\section{CAMRAD II}

The analytical results were obtained using the comprehensive analysis CAMRAD II. CAMRAD II is an aeromechanics analysis of rotorcraft that incorporates a combination of advanced technologies including multibody dynamics, nonlinear finite elements, and rotorcraft aerodynamics [3]. CAMRAD II has been used extensively for correlation of performance and loads measurements of the UH-60A in various flight conditions [7-10]. The aerodynamic model is based on second-order lifting line theory [11]. The blade section aerodynamic modeling in lifting line theory is unsteady, compressible, viscous flow about an infinite wing in a uniform flow consisting of a yawed freestream and wake-induced velocity. This problem is modeled within CAMRAD II as two-dimensional, steady, compressible, viscous flow (airfoil tables), plus corrections for swept and yawed flow, spanwise drag, unsteady loads, and dynamic stall. The wake modeling of lifting line theory is an incompressible vortex wake behind the lifting line with distorted geometry and rollup. The wake analysis calculates the rotor nonuniform induced velocities using rigid, prescribed, or free wake geometry. Two wake analyses are available in CAMRAD II: a rolled-up model and a multiple-trailer (with consolidation) model. The rolled-up wake model is based on the assumption that a tip vortex forms at the outboard blade tip. Because of its simplicity and efficiency, the rolled-up model has long been used for helicopter rotors. The multiple-trailer model has also been available and, with the consolidation feature, has been applied recently with success to tiltrotor and helicopter airloads calculations $[9,12]$.

The multiple-trailer model has the far wake trailed vorticity divided into several spanwise panels to provide more detailed structure for the inboard vorticity. This model has a discrete trailed vortex line emanating from each of the aerodynamic panel edges. The calculation of the free wake geometry includes the distortion of all of these trailed lines. With multiple far wake trailed vorticity panels, the trailed lines at the aerodynamic panel edges can be consolidated into rolledup lines using the trailed vorticity moment to scale the rate of roll-up. It is assumed that all the vorticity in each set eventually rolls up into a single vortex located at the centroid of the original vorticity distribution.

In this work, an isolated rotor was modeled as a flexible blade with 11 nonlinear finite elements and 21 aerodynamic panels. Detailed rotor control system geometry, stiffness, and lag damper were also incorporated. When not coupling to OVERFLOW, a dual-peak rigid wake model was used, except at $\mu=0.2$ where the free-wake model was used. The multiple-trailer with consolidation model was also used for the $\mu=0.2$ case and the results were compared with those with the baseline rolled-up wake model.

\section{OVERFLOW 2}

All Navier-Stokes Computational Fluid Dynamics (CFD) analyses presented herein were performed using OVERFLOW 2 version 2.2b [4]. OVERFLOW 2 is an overset, structured-mesh flow solver developed at NASA. For two decades the OVERFLOW solver has served to analyze a variety of rotorcraft under a wide range of flight conditions [13]. OVERFLOW 2 offers a wide variety of numerical schemes, turbulence models, and boundary conditions. For the present study, OVERFLOW 2 was run with 4th order central differencing and 4th order artificial dissipation in space. Time marching was performed using a 2nd order dual timestepping scheme. Turbulence was modeled near blade surfaces using the Spalart-Almaras one equation model with rotational corrections. The turbulence model was deactivated in regions one chord length or further from the rotor blades to reduce numerical dissipation of the wake. Blade surfaces were modeled as viscous, adiabatic walls; outer boundaries were modeled using a characteristic condition imposing freestream quantities. OVERFLOW 2 computes the flowfield by discretizing the Navier-Stokes equations on a series of overset, structured grids. Grids modeling the rotor blades were body-fitted and curvilinear. These grids, often called near-body grids, extended approximately one chord length from the blade surface. The near-body grids were nested within one or more grids, called off-body grids, which filled the space between the rotor and the boundary of the computational domain. The OVERFLOW 2 model included a notional hub, but the LRTA and wind tunnel struts were not modeled. All grids exchanged flow information in regions of overlap. The amount of this overlap was sufficient to support full 4th order accuracy at the boundaries.

The near-body grid representing the bulk of each rotor blade had an "O" topology with 157 points wrapping around the blade chordwise, 163 points along the blade span, and 75 points normal to the surface. The initial spacing at the blade surface had a $\mathrm{y}^{+}$value of 1 . This grid system is illustrated in Fig. 4(a). The near-body grid system (the hub and all four blades) contained approximately 11.6 million points. The off-body grid system used a series of ever larger Cartesian grids to create shells expanding outward from the near-body grid set. The grid point spacing within each shell is twice that of the shell immediately preceding it. The finest off-body grid had a spacing equivalent to 0.1 tip-chord lengths in all three directions. Seven such shells created a cubic computational domain spanning ten rotor radii in every 
direction. Domain boundaries in this scenario were set to a freestream characteristic condition thereby simulating a rotor operating in free air. Flow in the off-body grids was treated as inviscid and the turbulence model was deactivated. This off-body grid set consisted of 15.5 million grid points and is depicted in Fig. 4(b).

The near-body and off-body grid combination totals 27.1 million grid points. To reduce computation time, CFD simulations were run using a hybrid distributed/shared memory scheme with 160 Message Passing Interface (distributed memory) ranks and eight OpenMP threads (shared memory) per MPI rank for a total of 1280 parallel tasks. OVERFLOW 2 required approximately 31 minutes to advance the solution for this configuration $1 / 4$ of a rotor revolution using 1280 CPUs of an SGI Altix ICE computer.

\section{Coupled Analysis of CAMRAD II/OVERFLOW 2}

CAMRAD II uses a lower-fidelity aerodynamics model than that available in modern CFD codes, and most CFD codes lack the sophisticated Computational Structural Dynamics (CSD) and trim capabilities of comprehensive codes like CAMRAD II. Coupling a CFD code (e.g. OVERFLOW 2) to a comprehensive code (e.g. CAMRAD II) marries the strengths of the two approaches and produces the highestfidelity solution currently possible. For this study, coupling is achieved by alternate execution of OVERFLOW 2 and CAMRAD II. At the end of each code's turn to execute, it passes data to the next code. The data passed from OVERFLOW 2 to CAMRAD II is airload data integrated from its Navier-Stokes model of the UH-60 rotor. This airload data is used to augment CAMRAD II's internal aerodynamics model (which consists of airfoil tables and a lower-order wake model). At the end of its execution, CAMRAD II generates updated control positions and a description of how the blade deforms elastically as it revolves around the shaft. These quantities are used to give OVERFLOW 2's grids a realistic motion in response to the aerodynamic environment. This algorithm, called the delta coupling technique, was pioneered by Tung et al. [14] and implemented in OVERFLOW by Potsdam et al. [15] Significantly improved airloads prediction capability has been demonstrated for the UH-60A rotor in steady level flight conditions using this loosely coupled approach $[2,15,16]$. The CFD solution is advanced $1 / 4$ revolution during a coupling iteration because this allows each of the rotor's four blades to sweep through a full quadrant of the rotor disk. Taken in aggregate, the four blades thereby determine the airloads at every azimuth for every coupling iteration. Convergence of the coupling process was determined by monitoring blade airloads for periodicity. When the airloads did not vary significantly from one coupling iteration to the next, the solution was judged to be converged. For the present analysis, this generally occurred after 24 coupling iterations. Since OVERFLOW 2 was allowed to iterate for $1 / 4$ revolution between coupling exchanges, this equates to 6 full revolutions for the converged solution. A fully converged coupled solution required approximately 17 hours to compute on 1280 SGI Altix ICE processors.

For coupled analysis, CAMRAD II input was identical to uncoupled CAMRAD II input except the wake model was set to uniform inflow. The airload data passed to CAMRAD II accounted for both sectional aerodynamics and wake effects. The lifting-line aerodynamics and wake model were essentially replaced by airloads provided by OVERFLOW 2.

\section{Results and Discussion}

In this section, selected data from the wind tunnel test are compared with predictions from the two analysis methods. These test data include rotor trim, blade hinge motion, normal force, pitching moment, flap bending, chord bending, and torsion moments for both speed and thrust sweeps.

\section{Trim and blade motion for speed sweep}

Figures 5 and 6 show the calculated and measured blade pitch and flapping angles at the hinge at three different advance ratios; $\mu=0.2$ (Run 52, Point 20), $\mu=0.3$ (Run 52, Point 31), and $\mu=0.4$ (Run 52, Point 52) for the same $C_{L} / \sigma=0.09$. The trim solution for the speed sweep conditions in the analyses solves for the collective and cyclic angles required to match the measured thrust and hub moments with specified corrected shaft angle $\left(\alpha_{c}\right)$. The measured hinge motions were obtained from the $\mathrm{BMH}$ hardware and the values averaged over each of the four blades were used for comparison. The measured collective angles increase as the advance ratio increases and the analyses show the same trends but overpredict by about $1 \mathrm{deg}$ at all speeds investigated. Note that different scales are used for each plot. The calculations show good correlation of cyclic pitch angles, except lateral cyclic angle at $\mu=0.4$ where the analysis underpredicted by about $2 \mathrm{deg}$. The coupled analysis shows slightly better agreement with the measured cyclic angles compared to CAMRAD II alone.

The measured coning angles at the three different advance ratios are almost identical because lift is same for those conditions. The calculations show good agreement with the test data. The difference between the calculations and measured values are less than $0.2 \mathrm{deg}$. The difference between the two analyses is also small.

\section{Airloads for speed sweep}

Blade section normal force and pitching moment for the speed sweep are investigated in this section. Figure 7 shows nondimensional normal force at $92 \%$ radial station at three different advance ratios. At $\mu=0.2$, the measured normal force shows pulses due to blade vortex interaction (BVI) on both the advancing side and retreating side. The CAMRAD II 
analysis significantly overpredicts the magnitude of the downup impulse on the advancing side. The same overprediction was also observed with the UH-60A flight test correlation at $\mu=0.15$ [9]. This will be revisited in a later section. The coupled analysis accurately captures the magnitude of these pulses. At higher speed, the airloads on the blade tip region are generally characterized by negative lift at the end of the first quadrant and the beginning of the second quadrant [18] and both $\mu=0.3$ and 0.4 conditions show negative normal force at this radial location. This negative loading gets more severe as the advance ratio increases. The magnitude of the normal force is reasonably well predicted by CAMRAD II, but the phase angle correlation is fair to poor. The phase of the airloads from the coupled analysis is significantly improved over the CAMRAD II analysis. Small, high frequency oscillations in the test data in the first quadrant resulting from the wake interaction are also beginning to be captured in the coupled solution. Figure 8 shows nondimensional pitching moment at $92 \%$ radial station for the speed sweep. The CAMRAD II analysis shows a significant underprediction of advancing blade pitching moments at all advance ratios. Although there is an underprediction of peak-to-peak magnitude and an exaggerated dynamic stall cycle in the fourth quadrant at $\mu=0.4$, the coupled analysis significantly improves the overall correlation compared to the comprehensive code alone. especially the moment pulse due to BVI is better captured.

\section{Structural loads for speed sweep}

Figures 9 through 12 compare the calculated and measured structural loads for the speed sweep. Figure 9 shows oscillatory flap bending moment at $40 \%$ radial station. Steady values were removed from both test data and analyses. The CAMRAD II analysis showed poor correlation at $\mu=0.2$ due to the overprediction of the magnitude of the down-up normal force impulse on the advancing side as shown in Fig. 7(a). The CAMRAD II calculated flap bending moments show fair to good correlation on magnitude at $\mu=0.3$ and 0.4 , but the phase differs significantly from the measurements. The coupled analysis shows better prediction capability than the CAMRAD II analysis. Waveform and phase correlation is improved in the first and second quadrants by the coupled analysis.

Figure 10 shows oscillatory chord bending moment at $40 \%$ radial station. The measurements show significant high frequency content unlike flap bending moments. Both analyses underpredict the magnitude. Although the coupled analysis shows much stronger high frequency content than the comprehensive analysis, especially at $\mu=0.2$ and 0.3 , the peak-to-peak magnitude is significantly underpredicted and phase is not well captured.

Figure 11 shows oscillatory torsion moment at $40 \%$ radial station. Correlation is poor at $\mu=0.2$ for both analyses.
However both analyses, in general, show reasonably good correlation at higher advance ratios although peak-to-peak magnitude is underpredicted. The coupled analysis improves phase in the first and second quadrants and waveform in the third and fourth quadrants. The sharp drop around 250-deg azimuth is not well predicted even with the coupled analysis.

Figure 12 shows oscillatory pitch link loads. The measured pitch link loads show similar waveform as the torsion moments at $\mathrm{r} / \mathrm{R}=0.4$ on the advancing side, but much larger variation on the retreating side. The magnitude is well predicted in the first and second quadrants by both analyses, especially at $\mu=0.3$ and 0.4 , but the correlation is poor in the third and fourth quadrants. Although detailed shapes are better predicted by the coupled analysis, the sharp drop around 250-deg azimuth is not captured even with the coupled analysis.

Figure 13 compares harmonic magnitude of flap bending, chord bending, and torsion moments along the blade span for $\mu=0.3$. Note that different scales are used for each harmonic. The coupled analysis shows better agreement with the measured data on the $2 / \mathrm{rev}$ flap bending moment and most of the 4 and 5/rev moments. Neither analysis does well for the 1,2, and 3/rev chord bending moment. Even though a nonlinear lag damper model was used, both analyses significantly underpredict the magnitude of chord bending moment.

\section{Trim and blade motion for thrust sweep}

Figures 14 and 15 show the calculated and measured blade pitch and flapping angles at the hinge at two different thrust levels; $C_{T} / \sigma=0.06$ (Run 45, Point 30) and $C_{T} / \sigma=0.1255$ (Run 45, Point 38) for the same $\mu=0.3$. The trim solution for the CAMRAD II was solved for the controls that produced rotor thrust and hub moments to match the measured values, with the rotor shaft angle of attack fixed at the measured values with correction. It should be noted that the measured hub moments are very small as zero hub moments were sought during the test. For the coupled analysis, a different trim strategy was used. First, baseline case was selected at $C_{T} / \sigma=0.08$ and the simulation was trimmed to match the measured thrust. Collective deltas were derived from test data relative to this baseline and then applied to the simulated baseline to produce the remaining target points. This resulted in $C_{T} / \sigma=0.0591$ and 0.1243 , respectively. For the maximum thrust condition, the estimated thrust is about $0.96 \%$ lower than the measured value, with collective of $13.34 \mathrm{deg}$.

For the $C_{T} / \sigma=0.06$ case, the coupled analysis shows slightly better collective and coning angle correlation with the measured data than the comprehensive analysis, but both analyses overpredict them. For the highest thrust condition, although collective is still about $1.5 \mathrm{deg}$ higher than the measured value, the coupled analysis shows excellent agreement of coning angle. 


\section{Airloads for thrust sweep}

Figure 16 shows nondimensional section normal force at 92\% radial station for thrust sweep. The measured data show negative normal force at the end of first quadrant and beginning of second quadrant for both thrust levels. The measured normal force at $C_{T} / \sigma=0.06$ shows BVI-induced pulses in the first quadrant, which is similar to that observed at lower advance ratio (Fig. 7(a)). At higher thrust, the measured data show two stall events in the fourth quadrant. At $C_{T} / \sigma=0.06$ condition, the coupled analysis captures the BVI-induced normal force variation in the first quadrant and significantly improves phase correlation on the advancing side. At $C_{T} / \sigma=0.1255$ condition, the coupled analysis shows good correlation on the retreating side, capturing the large normal force fluctuations in the fourth quadrant due to dynamic stall events. However, the coupled analysis shows a large normal force variation in the first quadrant and this (along with pitching moment) has an important influence on high frequency structural loads that will be discussed later.

Figure 17 shows nondimensional section pitching moment at 92\% radial station for the thrust sweep. The CAMRAD II analysis again shows a significant underprediction of advancing blade pitching moments. It should be noted that the CAMRAD II analysis did not use a dynamic stall model and thus there is no high frequency pitching moment variation at $C_{T} / \sigma=0.1255$. The coupled analysis significantly improved the correlation by capturing BVI-induced high frequency impulse in the first quadrant at $C_{T} / \sigma=0.06$ and the magnitude and phase of the two stall events in the fourth quadrant at the deeply stalled condition $\left(C_{T} / \sigma=0.1255\right)$. Similar to the normal force, the coupled analysis shows a pitching moment pulse in the first quadrant, which was not observed in the measured data.

\section{Structural loads for thrust sweep}

Figures 18 through 21 show structural loads correlation for the thrust sweep. The coupled analysis shows much better flap bending moment correlation, as shown in Fig. 18, by improving both magnitude and phase correlation with the measured data. For the chord bending moment shown in Fig. 19, both analyses underpredict the magnitude at $C_{T} / \sigma=0.06$, although the coupled analysis shows slightly better peak-to-peak magnitude. However, the coupled analysis shows significant $5 / \mathrm{rev}$ chord bending moment. This overprediction of the $5 / \mathrm{rev}$ harmonic is also observed in the torsion moment and pitch link loads shown in Figs. 20 and 21, respectively. This can be explained from a coupled aerodynamic/structural dynamics point of view. Normal force and pitching moment pulses in the first quadrant, combined with two dynamic stall cycles in the fourth quadrant, generate a strong 5/rev forcing function. As the blade 2 nd chord frequency is about $4.69 / \mathrm{rev}$ and first torsion frequency is about $4.53 / \mathrm{rev}$, the structural responses to the 5/rev airloads are magnified. This will be further examined in a later section.

\section{Effect of wake modeling at low speed}

The CAMRAD II analysis significantly overpredicted the magnitude of the down-up impulse on the advancing side at $\mu=0.2$ as shown in Fig. 7(a). A similar trend was also observed with UH-60A flight test correlation at $\mu=0.15$ [9] and better correlation was obtained using a free wake geometry calculation method that combined the multipletrailer wake with a simulation of the tip vortex formation process (consolidation). Calculations are carried out with the multiple-trailer wake with consolidation in order to identify whether the same improvement can be obtained at this low speed condition. Figure 22 shows section normal force and oscillatory flap bending moment correlation. The multiple trailer with consolidation model reduced the magnitude of the pulse due to BVI compared to the baseline rolled-up wake on the advancing side and, thus, improved the normal force correlation. A slight improvement is also observed on the front of the rotor disk. A similar improvement is obtained for the flap bending moment using the prediction with the multiple trailer and consolidation model. This prediction is very close to the coupled analysis.

\section{Structural loads calculation with prescribed measured airloads}

This section investigates the structural loads calculated from prescribed measured airloads (normal force, chord force, and pitching moment) using CAMRAD II alone. Good correlation between prediction and test depends on three factors: accurate airloads measurement, accurate structural loads measurement, and accurate structural model. Prescribing measured airloads permits validation of the structural model without error associated with aerodynamic modelings. This approach has been proved successful for the correlation with the NASA/Army UH-60A Airloads Program flight test data [19]. This study found the present structural model to be adequate.

Structural loads are calculated for both $\mu=0.3$ and 0.4 and the results are compared with the measurements and the coupled analysis predictions as shown in Figs. 23 and 24. Good agreement between calculations and measurement is obtained for flap bending moment for both cases. Underprediction is observed for chord bending moment at $\mu=0.3$, but excellent correlation is obtained at $\mu=0.4$. Torsion moment correlation is poor. There are strong 5/rev responses, which were not observed with either CAMRAD II alone or coupled analysis. In order to understand poor correlation of torsion moments, section normal force and pitching moment near the blade tip area are compared in Figs. 25 and 26. Although there are pressure transducers installed at $r / R=0.965$, airloads are not available at that radial location due to malfunction. One obvious outlier is $r / R=0.99$ where there is a significant mean value change and a big hump in pitching moment in the first quadrant for both advance ratios. This may be the cause of a strong 5/rev response in the torsion moments. Without 
measurements at $\mathrm{r} / \mathrm{R}=0.965$, this is the only airload data available acting on the swept tip. Because of the sweep, these airloads will significantly twist the blade. The same issue was observed at other advance ratios (not shown in this paper) and thus assessment of structural loads with prescribed measured airloads was not successfully carried out in full. Detailed examination of pressures at this radial location is warranted.

\section{Parametric studies of maximum thrust condition}

At the high thrust condition, the predicted airloads featured large, first quadrant normal force and pitching moment variations that appear to stimulate a strong 5/rev structural response. These phenomena are not present in the measured data. In an effort to understand the cause of these mispredictions the effects of collective and pitch link stiffness on airloads and structural loads are investigated. All the results shown in Figs. 27 through 29 were obtained with the coupled analysis. Figure 27 shows the calculated pitch angle at the maximum thrust. Figure 27(a) is the result of two different collective angles, $11.88 \mathrm{deg}$ and $14.85 \mathrm{deg}$. The $11.88 \mathrm{deg}$ was chosen to closely match with the measured collective and $14.85 \mathrm{deg}$ was chosen because it is roughly the same collective change from the baseline collective of $13.34 \mathrm{deg}$. The lower collective produced $C_{T} / \sigma=0.1187$ which is $5.42 \%$ lower than the measured value and the higher collective produced $C_{T} / \sigma=0.1273$ which is $1.43 \%$ higer. Larger collective required larger cyclic pitch angles to trim the rotor. Another variation, shown in Fig. 27(b), is pitch link stiffness with the baseline collective. The baseline pitch link stiffness value of $1090 \mathrm{ft}-\mathrm{lb} / \mathrm{deg}$, which is widely used for previous UH-60A analyses, was determined experimentally from direct measurements of the aircraft hardware by Kufeld and Johnson [20]. The soft value was obtained from the study by Shanley [21] under a NASA contract.

Figure 28 shows section airloads due to the parametric changes. The lower collective (matching measured collective) reduces the normal force and pitching moment pulse in the first quadrant, but underpredicts negative normal force peak on the advancing side and misses the phase of two pitching moment peaks on the retreating side. The higher collective generates advancing blade stall spikes and degrades correlation. The soft pitch link improves correlation by reducing the normal force and pitching moment pulse in the first quadrant and correcting normal force phase on the advancing side. None of these improves pitching moment correlation across the front of the rotor disk.

Figure 29 shows the effects of collective and pitch link stiffness on structural loads. Both lower collective and soft pitch link stiffness cases show slightly better correlation than the baseline results. The higher collective generates substantially larger 5/rev structural response. This is clearly illustrated by the $5 / \mathrm{rev}$ torsion moment harmonic correlation shown in Figs. 29(e) and 29(f). As the blade 2nd chord frequency is about 4.69/rev and first torsion frequency is about $4.53 / \mathrm{rev}$, the structural responses to the 5/rev airloads due to dynamic stall are magnified. In this case, the soft pitch link moves the first torsion frequency from $4.53 / \mathrm{rev}$ to $4.0 / \mathrm{rev}$ and prevents this excitation.

Near the rotor thrust limit the measured thrust showed reduced sensitivity to collective change. The higher collective case overpredicted the rotor lift by just $1.43 \%$ but produced a dynamic stall event on the advancing side and significantly larger 5/rev structural responses.

\section{Conclusions}

Wind tunnel measurements of the rotor trim, blade airloads, and structural loads of a full-scale UH-60A Black Hawk main rotor are compared with calculations obtained using the comprehensive rotorcraft analysis CAMRAD II alone and a coupled CAMRAD II/OVERFLOW 2 analysis. A speed sweep at constant lift up to an advance ratio of 0.4 and a thrust sweep at constant speed into deep stall are investigated.

From this study the following conclusions were obtained:

\section{Speed sweep}

1) The comprehensive analysis with lifting-line aerodynamics significantly overpredicted the magnitude of the down-up impulse in normal force on the advancing side at low advance ratio. However, the magnitude of the normal force is reasonably well predicted at higher advance ratios. Better normal force correlation is obtained at low advance ratio by using a free-wake geometry calculation method that combines the multiple-trailer wake with a simulation of the tip vortex formation process (consolidation).

2) The coupled analysis shows significant improvement over comprehensive analysis. Pitching moment magnitudes are better captured in the coupled solutions. The shape of the airloads curves is better predicted with the coupled analysis.

3) The comprehensive analysis shows reasonably good flap bending and torsion moment correlation but significantly underpredicts the magnitude of chord bending moment. The coupled analysis improves waveforms and phase of flap bending and torsion moment but does not improve the chord bending moment much.

4) Structural loads were calculated by prescribing measured airloads. Strong 5/rev torsion moment, which were not observed in the test, were predicted and attributed to the lack of measurements at $\mathrm{r} / \mathrm{R}=0.965$ and anomaly, especially in pitching moment, at $\mathrm{r} / \mathrm{R}=0.99$.

\section{Thrust sweep}

5) The coupled analysis again shows significant improvement in the airloads predictions, especially capturing the magnitude 
and phase of the two stall events in the fourth quadrant at the deeply stalled condition.

6) Accurate analysis of the maximum thrust condition is challenging because large collective variation produces small thrust change. The higher collective case, although overpredicting lift by just $1.43 \%$, produces a dynamic stall event on the advancing side and significantly larger 5/rev structural responses.

7) As the blade 2nd chord frequency is about $4.69 /$ rev and first torsion frequency is about $4.53 / \mathrm{rev}$, the structural responses to the 5/rev airloads due to dynamic stall are magnified. In this case, a soft pitch link which moves the first torsion frequency from $4.53 / \mathrm{rev}$ to $4.0 / \mathrm{rev}$ improves the overall structural loads correlation.

\section{References}

[1] Norman, T. R., Shinoda, P. M., Peterson, R. L., and Datta, A., "Full-scale Wind Tunnel Test of the UH-60A Airloads Rotor," American Helicopter Society 67th Annual Forum, Virginia Beach, VA, May 3-5, 2011.

[2] Romander, E., Norman, T. R., and Chang, I-C., "Correlating CFD Simulation With Wind Tunnel Test for the Full-Scale UH-60A Airloads Rotor," American Helicopter Society 67th Annual Forum, Virginia Beach, VA, May 3-5, 2011.

[3] Johnson, W., "Technology Drivers in the Development of CAMRAD II," American Helicopter Society Aeromechanics Specialist Meeting, San Francisco, CA, January 1994.

[4] Buning, P. G., Gomez, R. J., and Scallion, W. I., "CFD Approaches for Simulation of Wing-Body Stage Separation," AIAA-2004-4838, AIAA 22nd Applied Aerodynamics Conference, Providence, RI, August 1619, 2004.

[5] Bousman, W. G., and Kufeld, R. M., "UH-60A Airloads Catalog," NASA TM-2005-212827, August 2005.

[6] McHugh, F. J., Clark, R., and Solomon, M., "Wind Tunnel Investigation of Rotor Lift and Propulsive Force at High Speed - Data Analysis," NASA CR 145217-1, October 1977.

[7] Yeo, H., Bousman, W. G., and Johnson, W., "Performance Analysis of a Utility Helicopter with Standard and Advanced Rotor,' Journal of the American Helicopter Society, Vol. 49, No. 3, July 2004, pp. 250270.

[8] Shinoda, P. M., Yeo. H., and Norman, T. R., "Rotor Performance of a UH-60 Rotor System in the NASA Ames 80 - by 120-Foot Wind Tunnel," Journal of the
American Helicopter Society, Vol. 49, No. 4, October 2004.

[9] Yeo, H., and Johnson, W., "Assessment of Comprehensive Analysis Calculation of Airloads on Helicopter Rotors," Journal of Aircraft, Vol. 42, No. 5, September-October 2005.

[10] Yeo, H., and Johnson, W., "Prediction of Rotor Structural Loads with Comprehensive Analysis," Journal of the American Helicopter Society, Vol. 53, No. 2, April 2008.

[11] Johnson, W., "Recent Developments in Rotary-Wing Aerodynamic Theory," AIAA Journal, Vol. 24, No. 8, 1986, pp. 1219-1244.

[12] Johnson, W., "Influence of Wake Models on Calculated Tiltrotor Aerodynamics," American Helicopter Society Aerodynamics, Acoustics, and Test and Evaluation Technical Specialist Meeting Proceedings, San Francisco, CA, January 2002.

[13] Potsdam, M., Strawn, R. C., and Meakin, R., "Dynamic Rotorcraft Applications Using Overset Grids," 31st European Rotorcraft Forum, Florence, Italy, September 13-15, 2005.

[14] Tung, C., Caradonna, F. X., and Johnson, W., "The Prediction of Transonic Flows on an Advancing Rotor," American Helicopter Society 40th Annual Forum, Arlington, VA, May 16-18, 1984.

[15] Potsdam, M., Yeo, H., and Johnson, W., "Rotor Airloads Prediction Using Loose Aerodynamic/Structural Coupling," Journal of Aircraft, Vol. 43, No. 3, MayJune 2006.

[16] Nygaard, T., Saberi, H., Ormiston, R. A., Strawn, R. C., and Potsdam, M., "CFD and CSD Coupling Algorithms and Fluid Structure Interface for Rotorcraft Aeromechanics in Steady and Transient Flight Conditions," American Helicopter Society 62nd Annual Forum, Phoenix, AZ, May 9-11, 2006.

[17] Yeo, H., Romander, E., and Norman, T. R., "Investigation of Rotor Performance and Loads of a UH-60A Individual Blade Control System," American Helicopter Society 66th Annual Forum, Phoenix, AZ, May 3-5, 2010.

[18] Hooper, W. E., "The Vibratory Airloads of Helicopter Rotors," Vertica, Vol. 8, No. 2, 1984.

[19] Ho, J. C., Yeo, H., and Ormiston, R. A., "Investigation of Rotor Blade Structural Dynamics and Modeling Based on Measured Airloads," Journal of Aircraft, Vol. 45, No. 5, September-October 2008. 
[20] Kufeld, R. M., and Johnson, W., "The Effects of Control System Stiffness Models on the Dynamic Stall Behavior of a Helicopter," Journal of the American Helicopter Society, Vol. 45, No. 4, October 2000.

[21] Shanley, J. P. "Validation of UH-60A CAMRAD/JA Input Model," SER 701716, Sikorsky Aircraft, November 1991. 


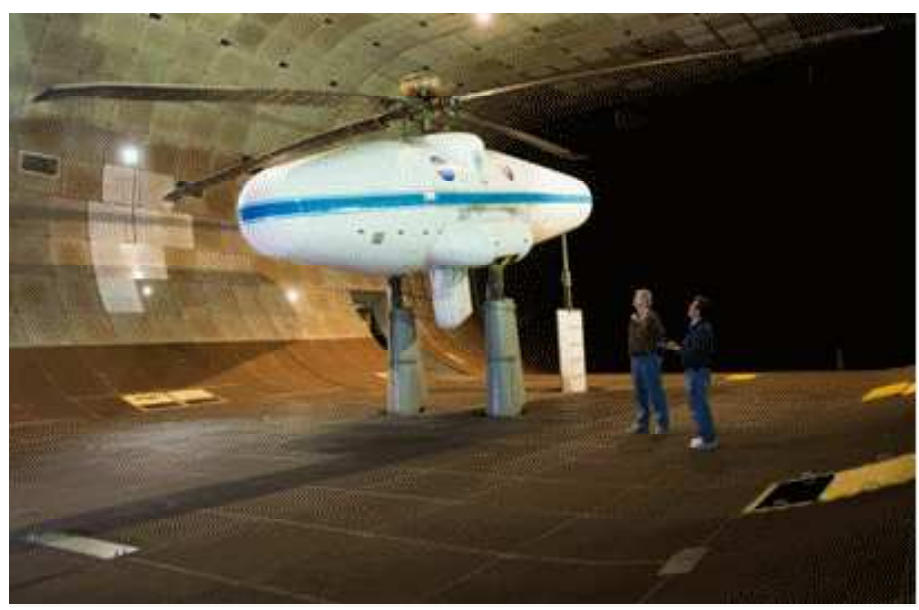

Fig. 1: UH-60A rotor system installed on the Large Rotor Test Apparatus in the NFAC 40- by 80-Foot Wind Tunnel.

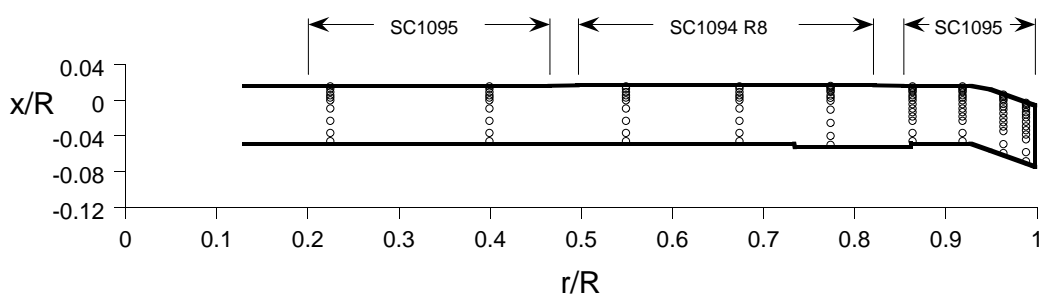

(a) Pressure transducer locations

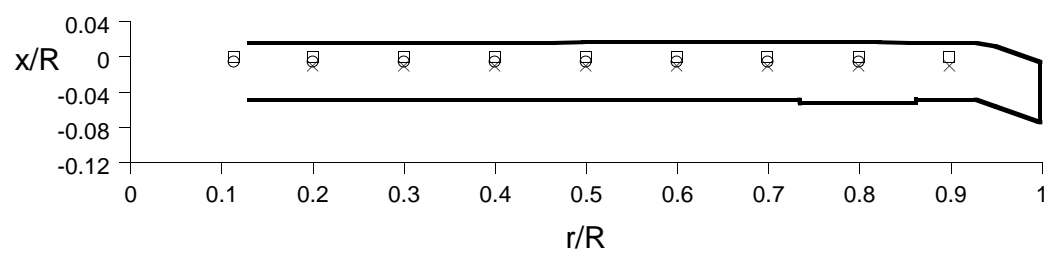

(b) Strain gauge locations ( $\square$ : flap bending, o: chord bending, and $\times$ : torsion moment)

Fig. 2: Blade planform with locations of pressure transducers and strain gauges. 


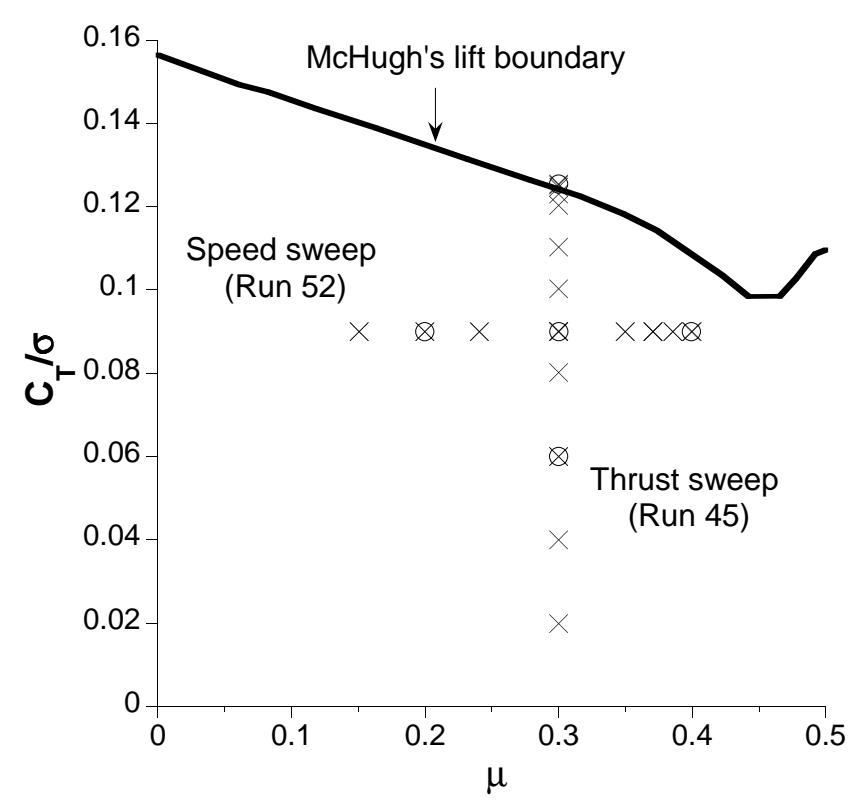

Fig. 3: Test conditions for speed and thrust sweep (circles represent test points investigated).

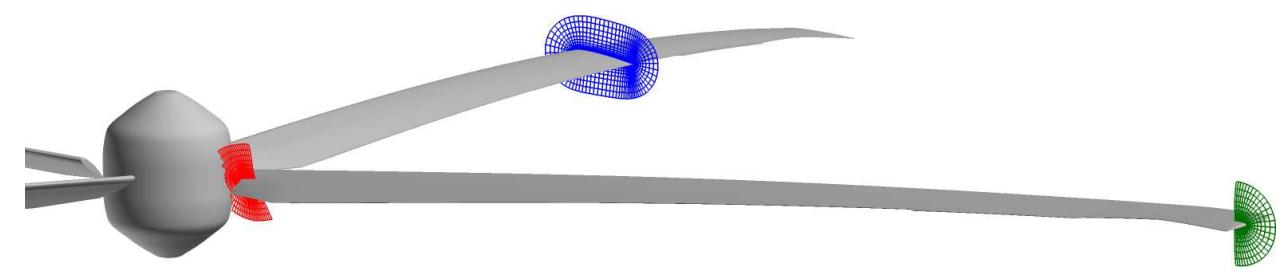

(a) Near-body grid system (not every point shown)

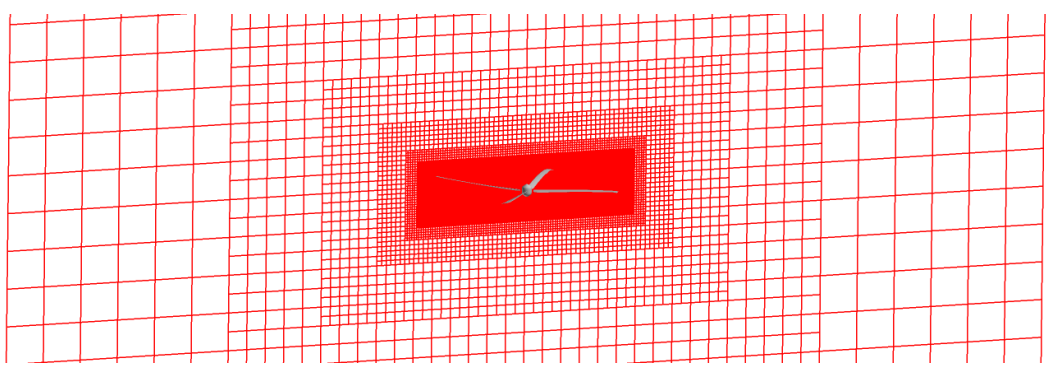

(b) Off-body grid system

Fig. 4: OVERFLOW 2 grid system. 


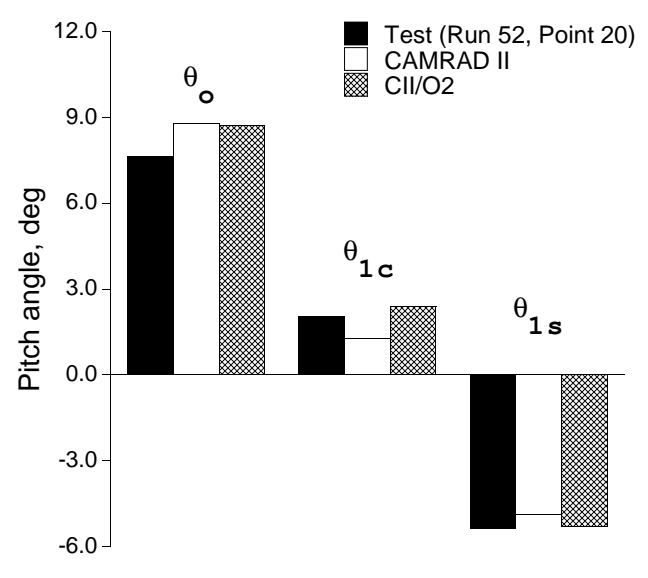

(a) $\mu=0.2, C_{L} / \sigma=0.09$

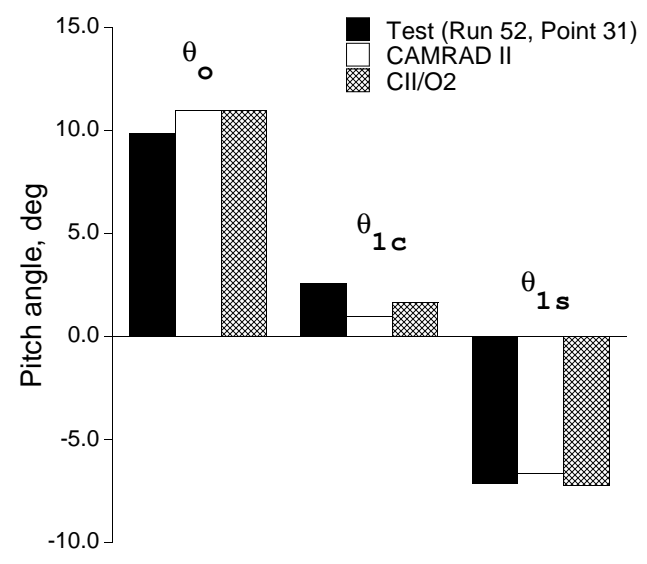

(b) $\mu=0.3, C_{L} / \sigma=0.09$

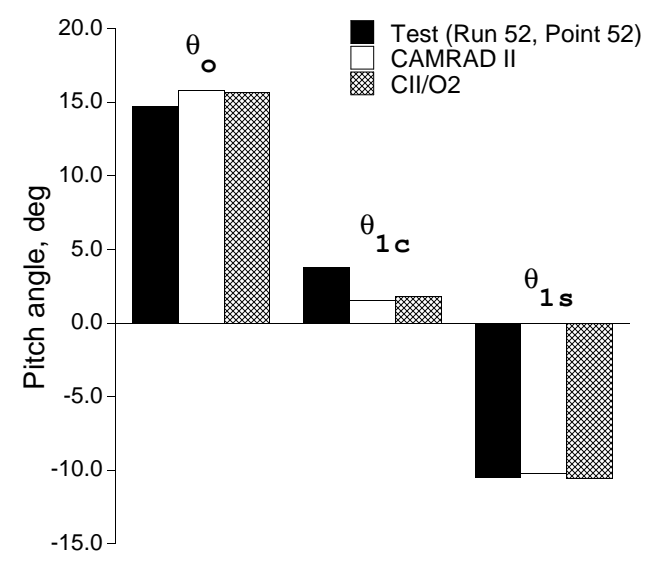

(c) $\mu=0.4, C_{L} / \sigma=0.09$

Fig. 5: Calculated and measured blade pitch angle for speed sweep.

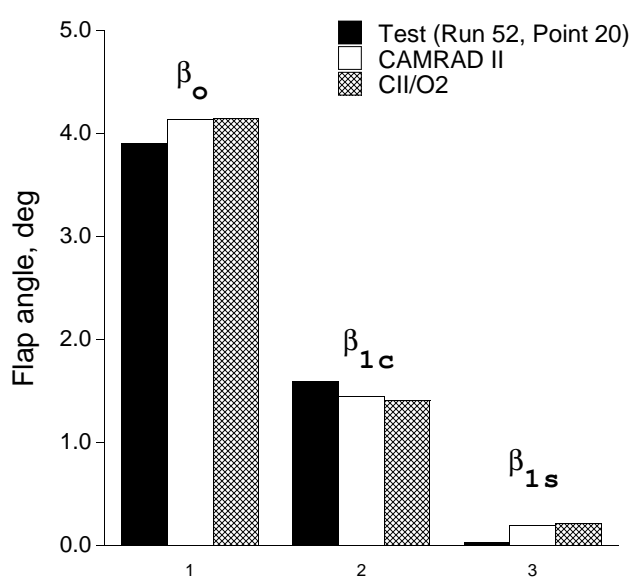

(a) $\mu=0.2, C_{L} / \sigma=0.09$

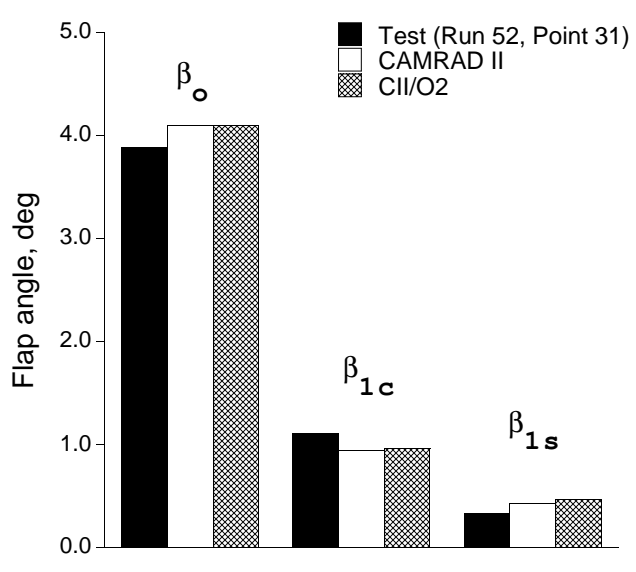

(b) $\mu=0.3, C_{L} / \sigma=0.09$

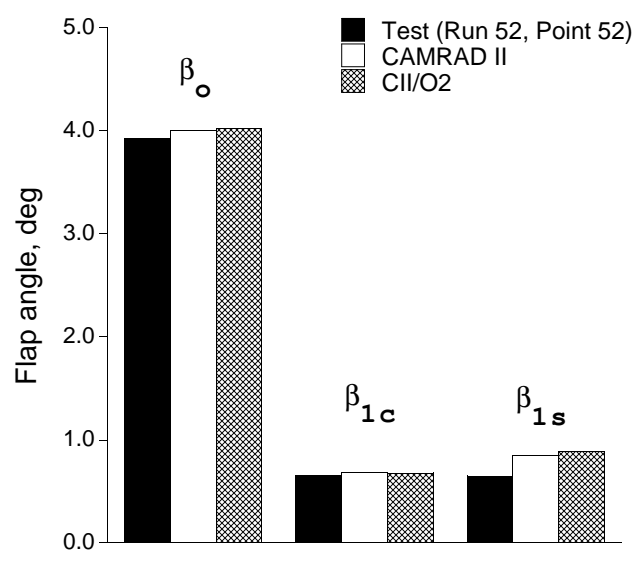

(c) $\mu=0.4, C_{L} / \sigma=0.09$

Fig. 6: Calculated and measured blade flap angle for speed sweep. 


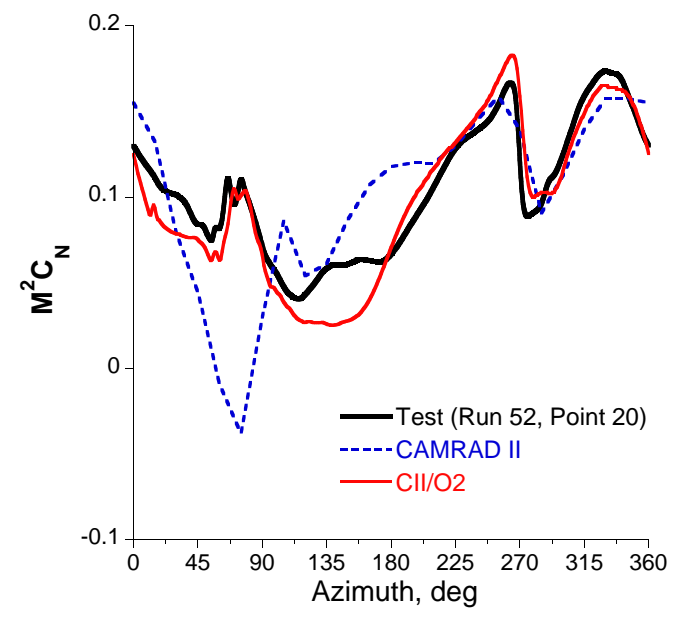

(a) $\mu=0.2, C_{L} / \sigma=0.09$

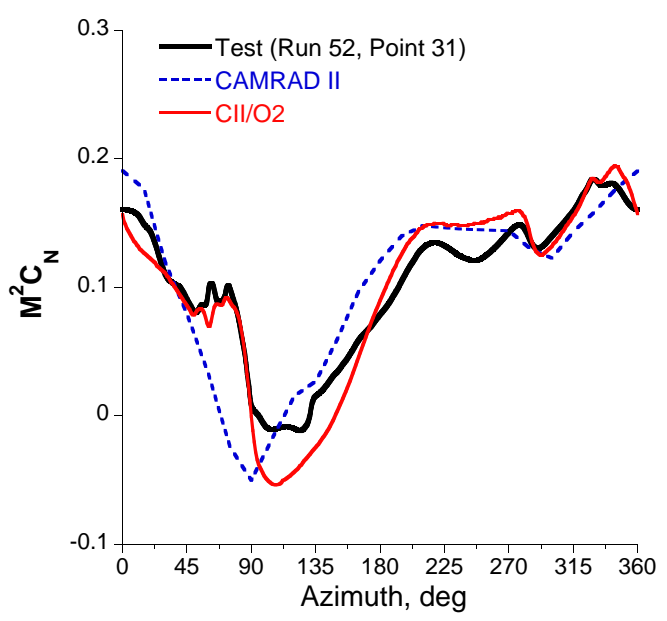

(b) $\mu=0.3, C_{L} / \sigma=0.09$

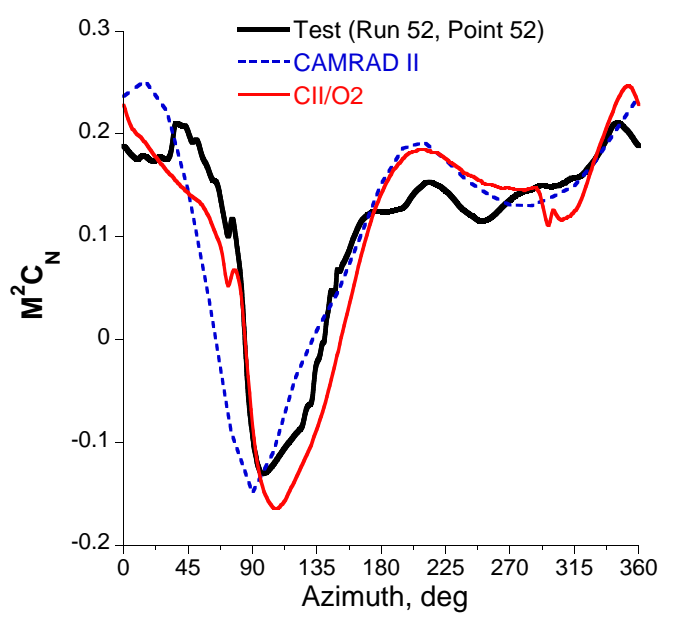

(c) $\mu=0.4, C_{L} / \sigma=0.09$

Fig. 7: Calculated and measured blade section normal force at $\mathrm{r} / \mathrm{R}=0.92$ for speed sweep.

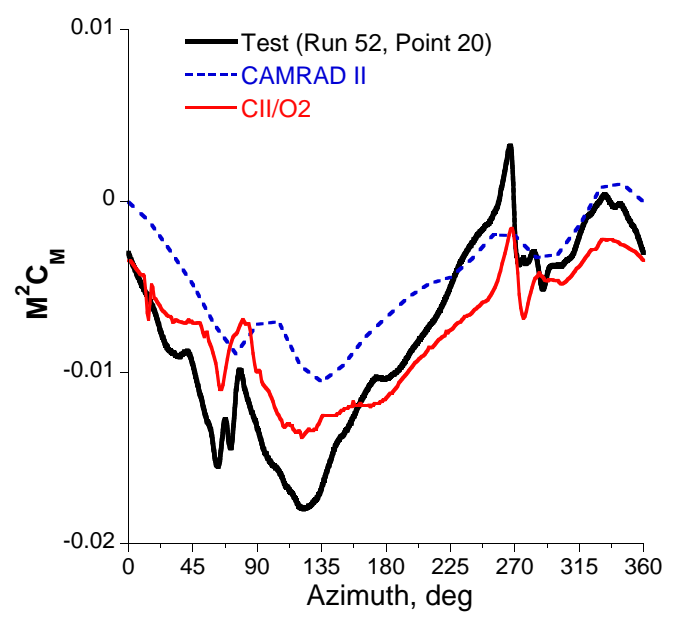

(a) $\mu=0.2, C_{L} / \sigma=0.09$

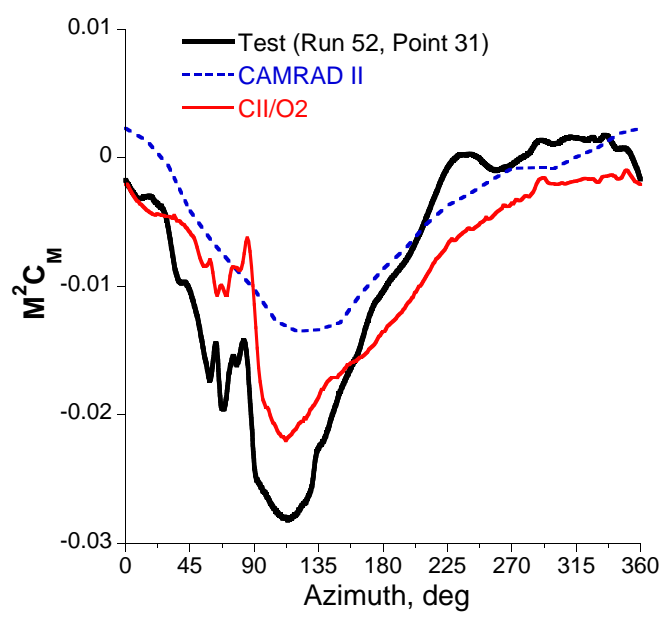

(b) $\mu=0.3, C_{L} / \sigma=0.09$

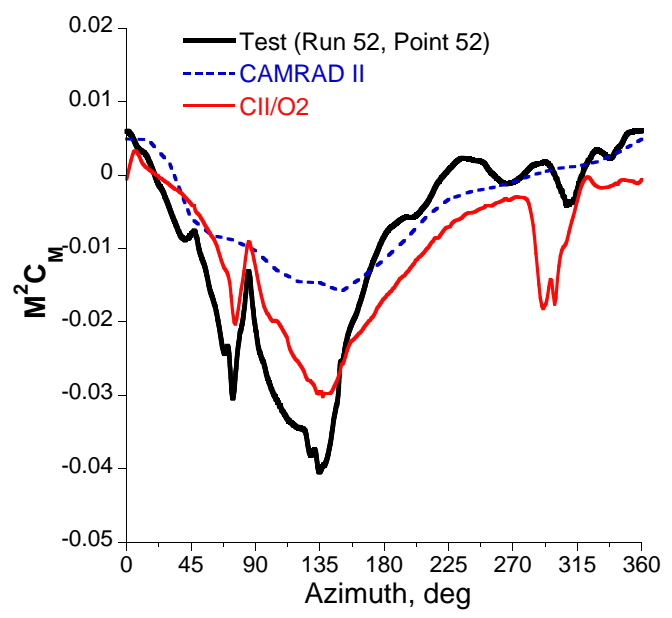

(c) $\mu=0.4, C_{L} / \sigma=0.09$

Fig. 8: Calculated and measured blade section pitching moment at $\mathrm{r} / \mathrm{R}=0.92$ for speed sweep. 


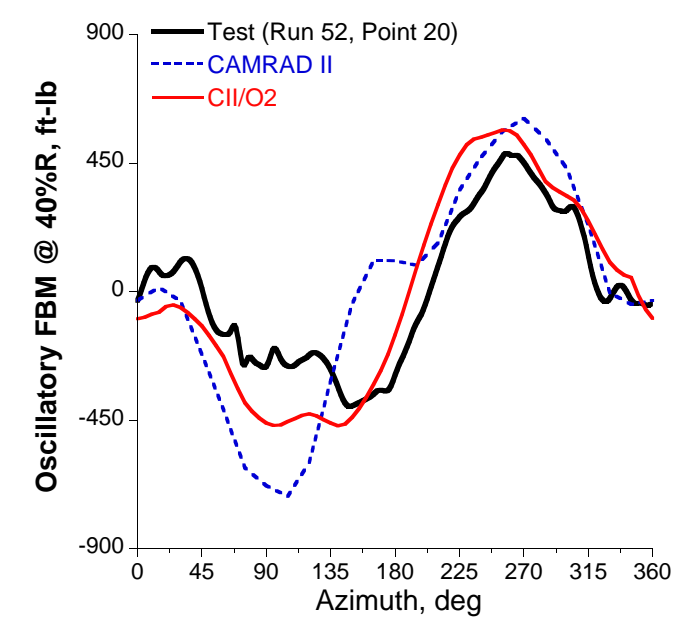

(a) $\mu=0.2, C_{L} / \sigma=0.09$

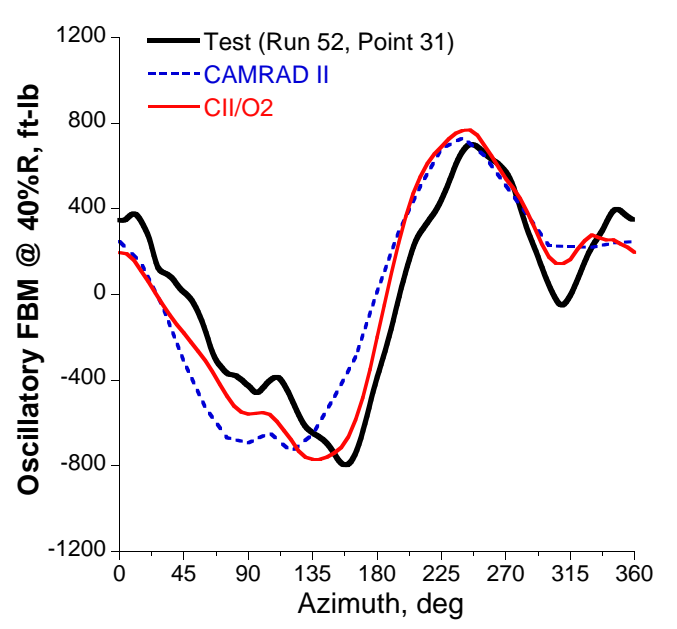

(b) $\mu=0.3, C_{L} / \sigma=0.09$

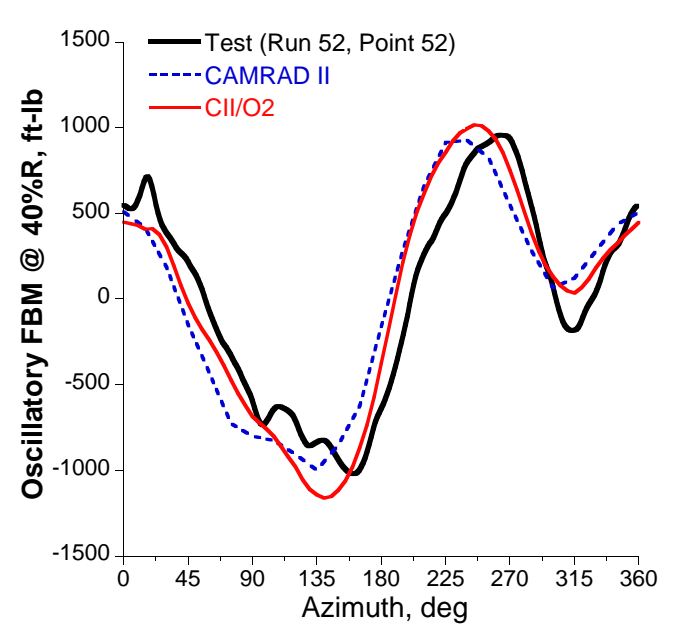

(c) $\mu=0.4, C_{L} / \sigma=0.09$

Fig. 9: Calculated and measured blade oscillatory flap bending moment at $\mathrm{r} / \mathrm{R}=0.4$ for speed sweep.

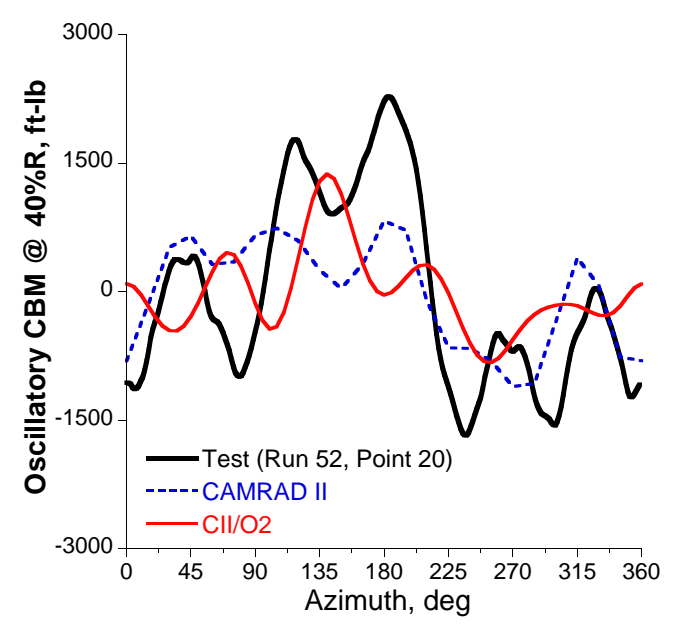

(a) $\mu=0.2, C_{L} / \sigma=0.09$

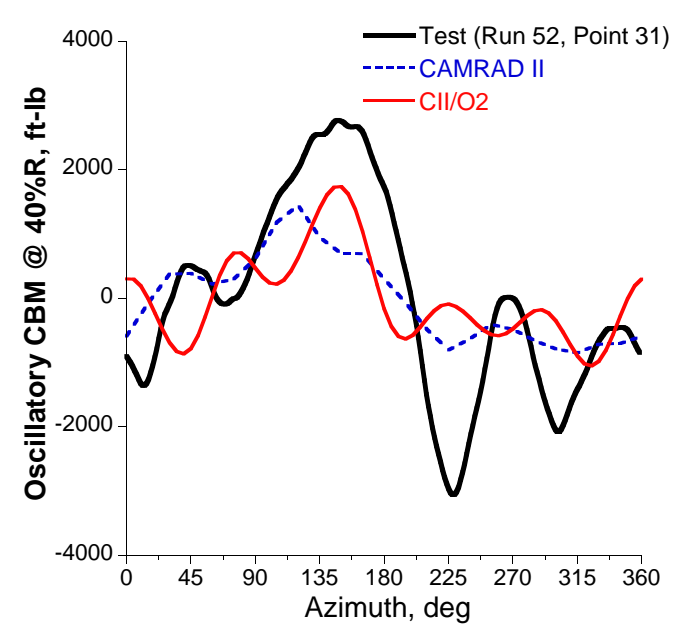

(b) $\mu=0.3, C_{L} / \sigma=0.09$

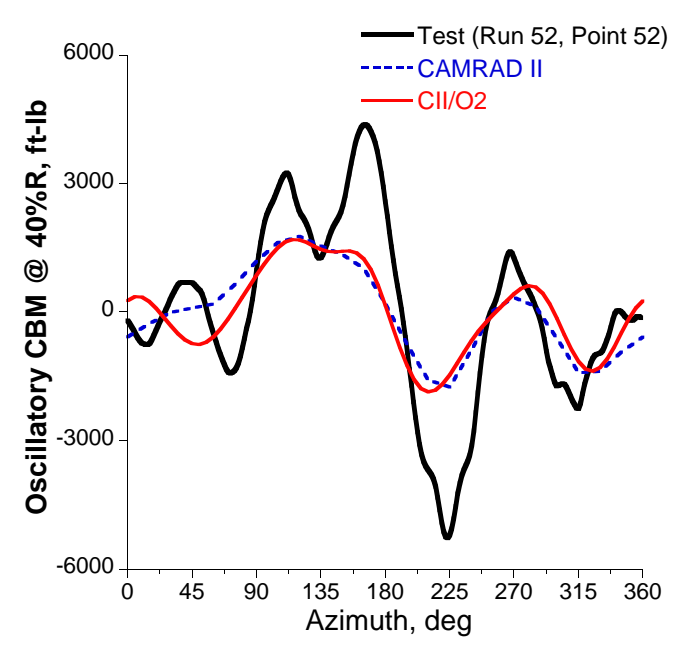

(c) $\mu=0.4, C_{L} / \sigma=0.09$

Fig. 10: Calculated and measured blade oscillatory chord bending moment at $\mathrm{r} / \mathrm{R}=0.4$ for speed sweep. 


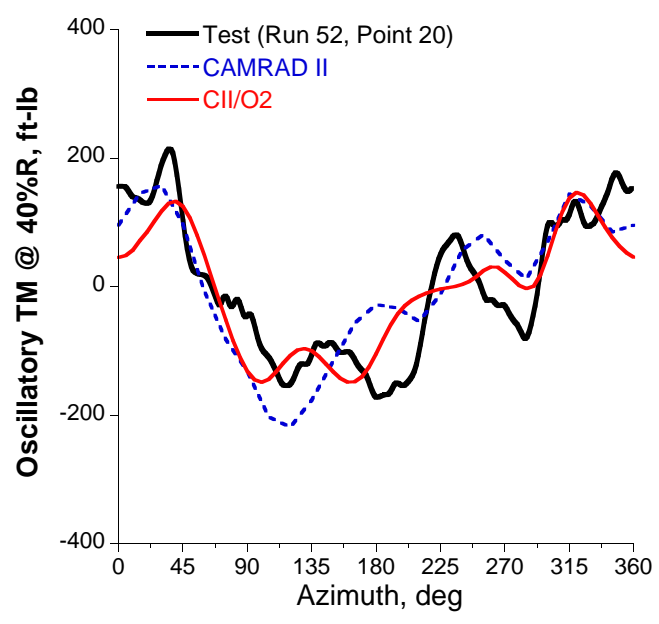

(a) $\mu=0.2, C_{L} / \sigma=0.09$

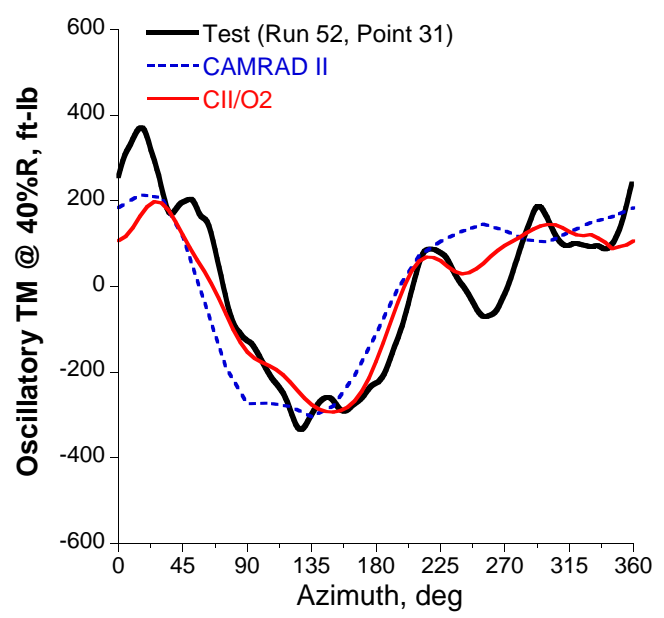

(b) $\mu=0.3, C_{L} / \sigma=0.09$

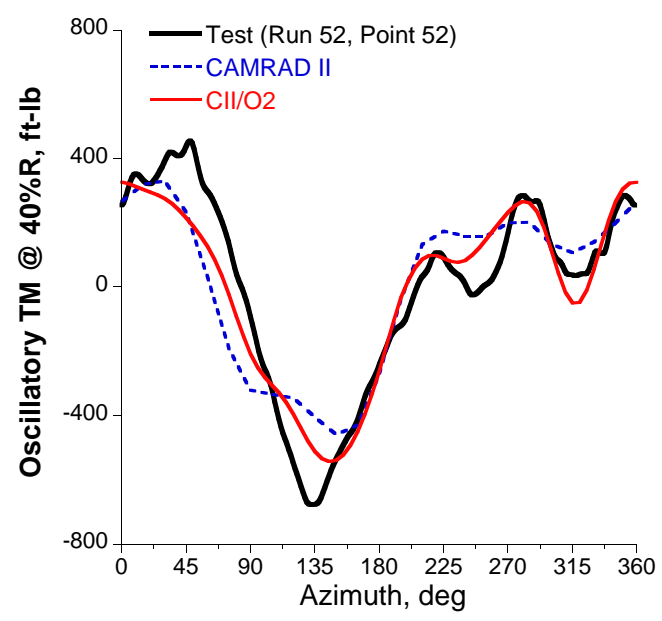

(c) $\mu=0.4, C_{L} / \sigma=0.09$

Fig. 11: Calculated and measured blade oscillatory torsion moment at $\mathrm{r} / \mathrm{R}=0.4$ for speed sweep.

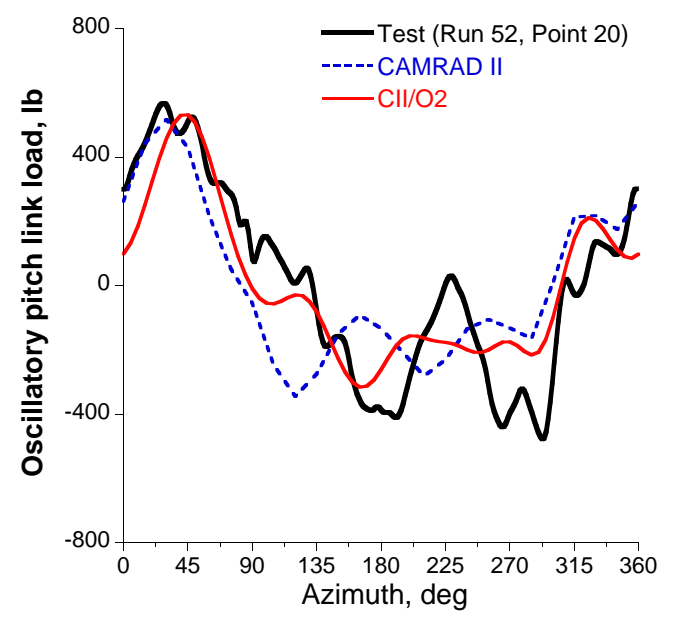

(a) $\mu=0.2, C_{L} / \sigma=0.09$

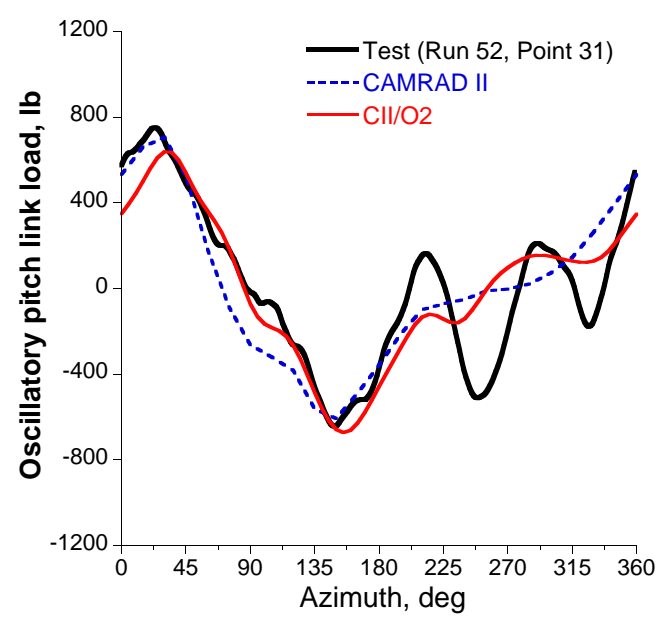

(b) $\mu=0.3, C_{L} / \sigma=0.09$

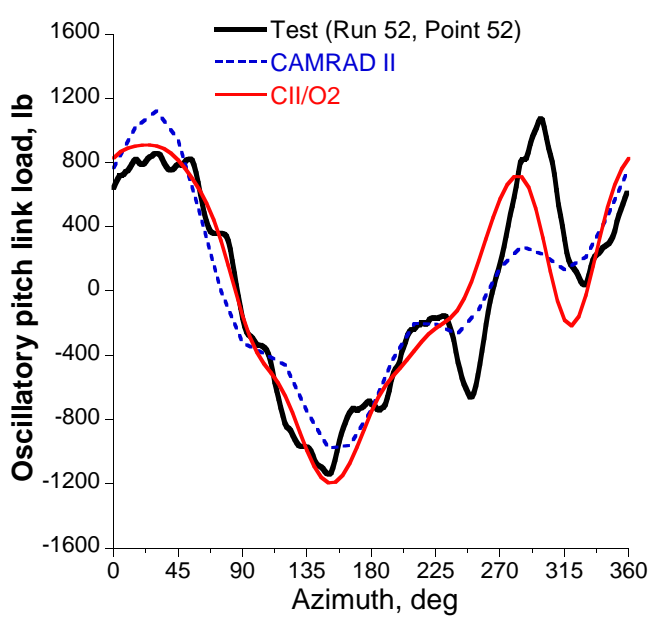

(c) $\mu=0.4, C_{L} / \sigma=0.09$

Fig. 12: Calculated and measured oscillatory pitch link load for speed sweep. 


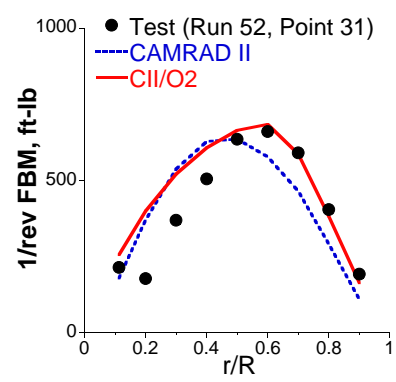

(a) 1/rev magnitude

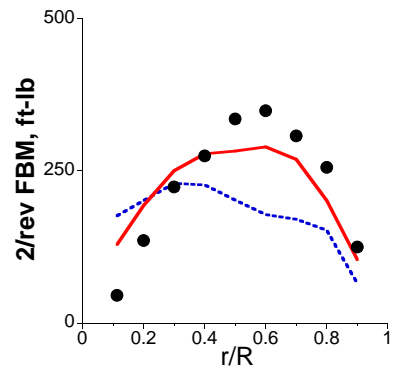

(d) $2 /$ rev magnitude

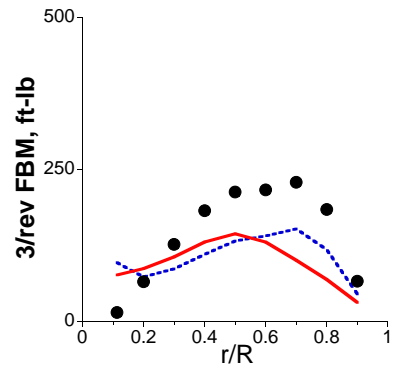

(g) 3/rev magnitude

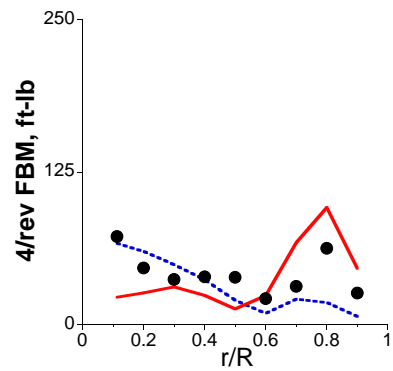

(j) 4/rev magnitude

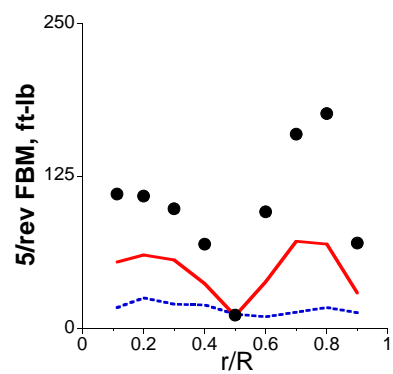

(m) 5/rev magnitude

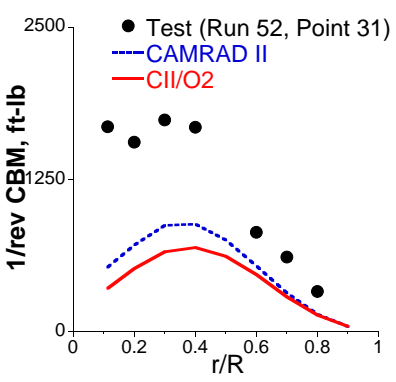

(b) $1 /$ rev magnitude

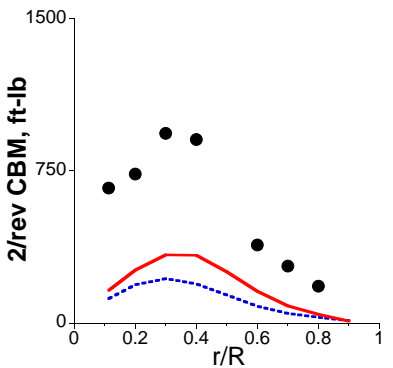

(e) $2 /$ rev magnitude

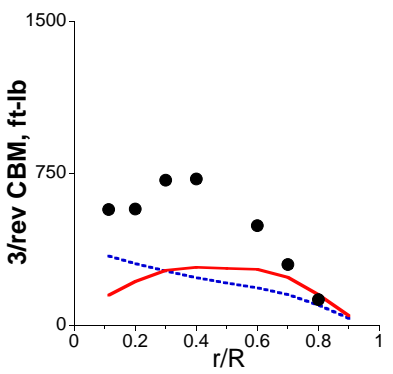

(h) $3 /$ rev magnitude

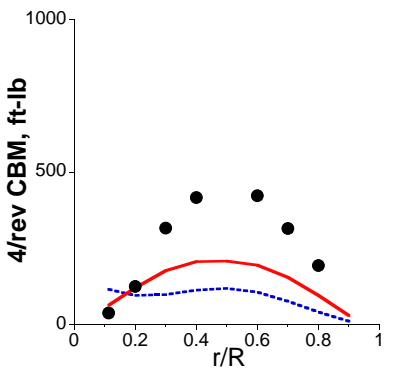

(k) 4/rev magnitude

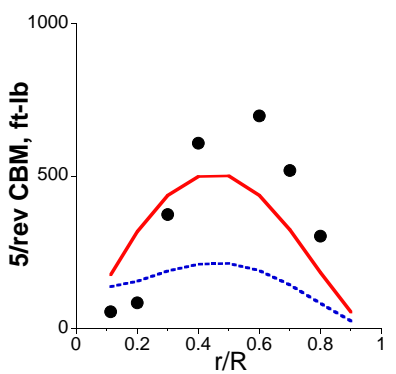

(n) $5 /$ rev magnitude

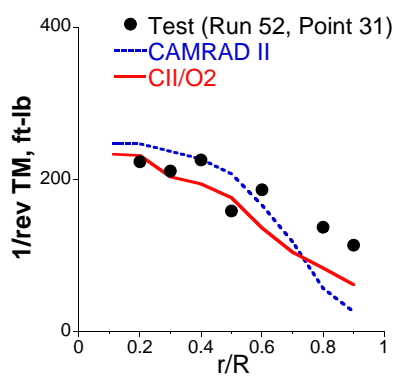

(c) $1 /$ rev magnitude

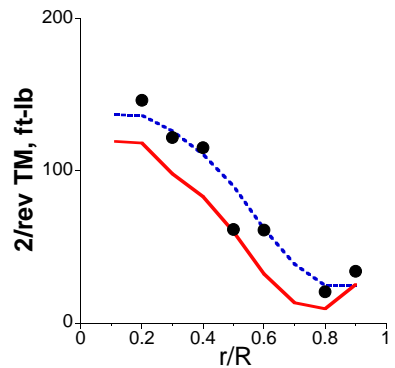

(f) $2 /$ rev magnitude

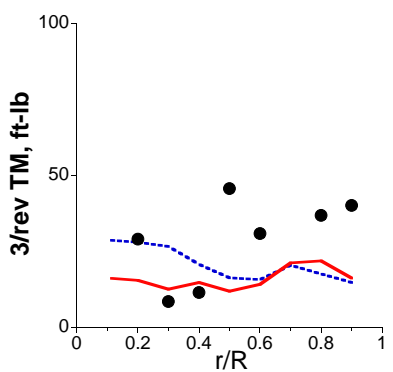

(i) 3/rev magnitude

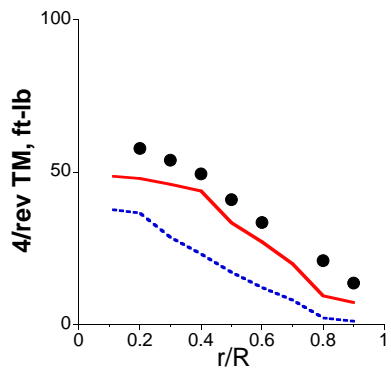

(1) $4 /$ rev magnitude

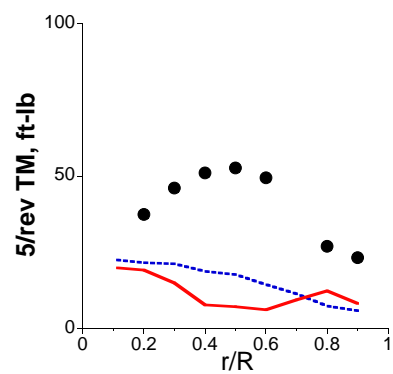

(o) $5 /$ rev magnitude

Fig. 13: Comparison of harmonic magnitude of calculated and measured structural loads, $\mu=0.3, C_{L} / \sigma=0.09$ 


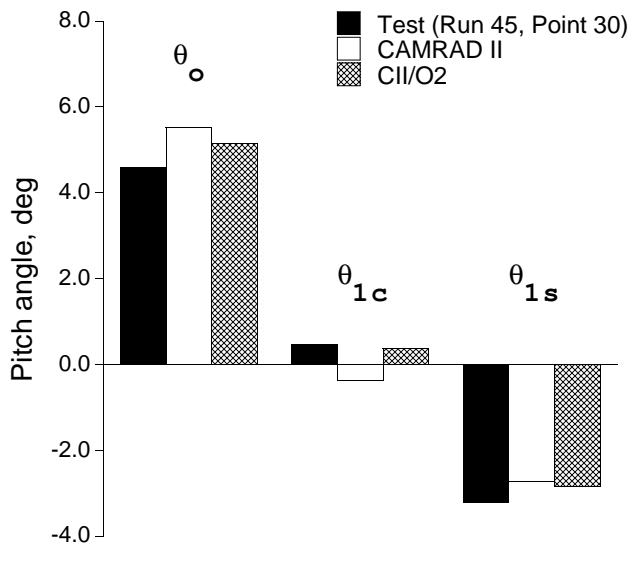

(a) $\mu=0.3, C_{T} / \sigma=0.06$

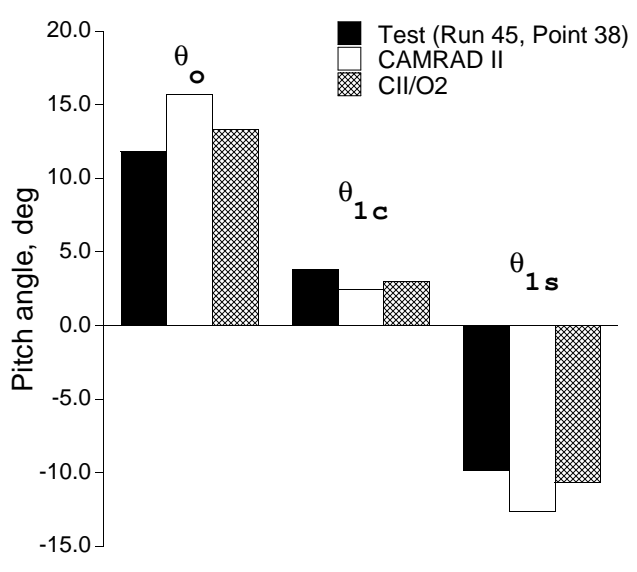

(b) $\mu=0.3, C_{T} / \sigma=0.1255$

Fig. 14: Calculated and measured blade pitch angle for thrust sweep.

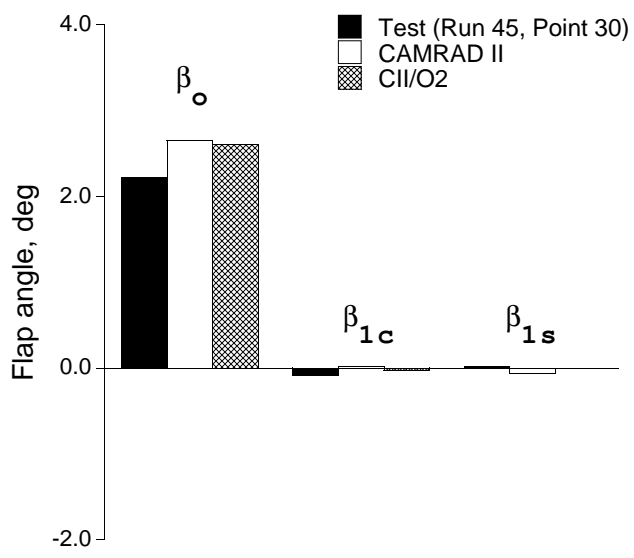

(a) $\mu=0.3, C_{T} / \sigma=0.06$

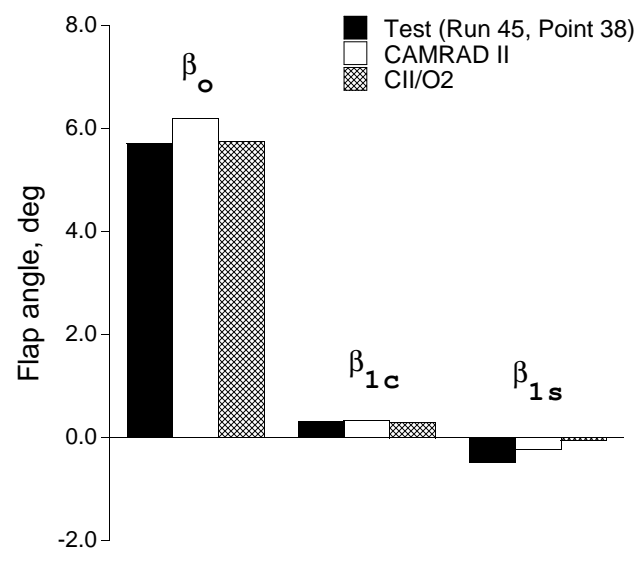

(b) $\mu=0.3, C_{T} / \sigma=0.1255$

Fig. 15: Calculated and measured blade flap angle for thrust sweep.

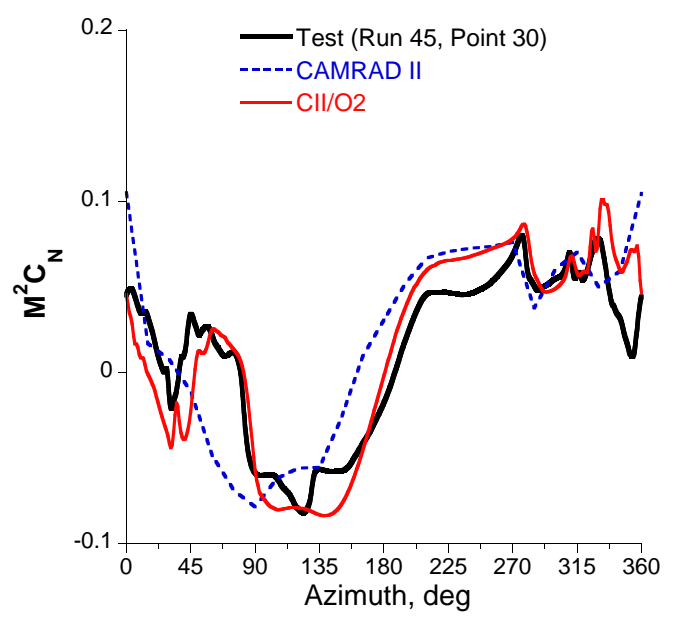

(a) $\mu=0.3, C_{T} / \sigma=0.06$

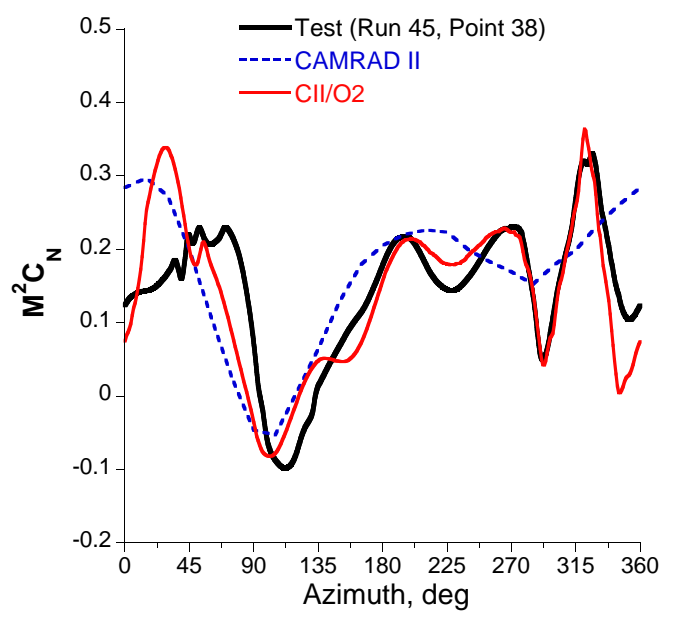

(b) $\mu=0.3, C_{T} / \sigma=0.1255$

Fig. 16: Calculated and measured blade section normal force at $\mathrm{r} / \mathrm{R}=0.92$ for thrust sweep. 


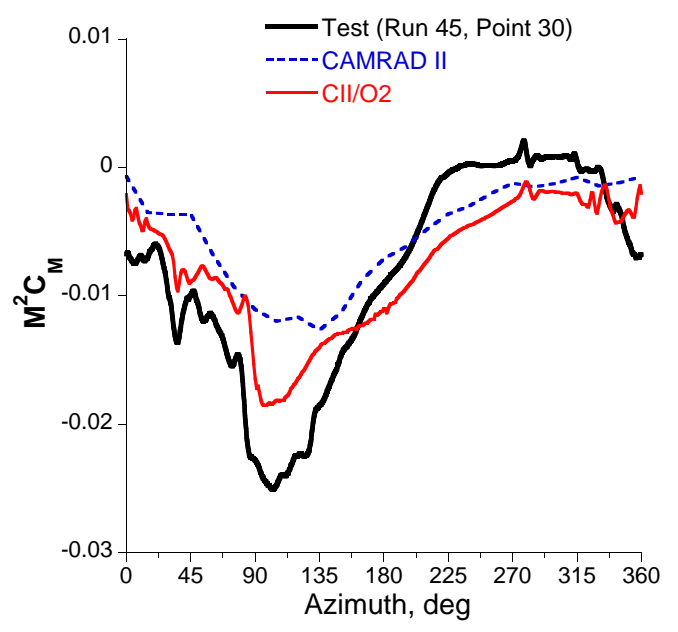

(a) $\mu=0.3, C_{T} / \sigma=0.06$

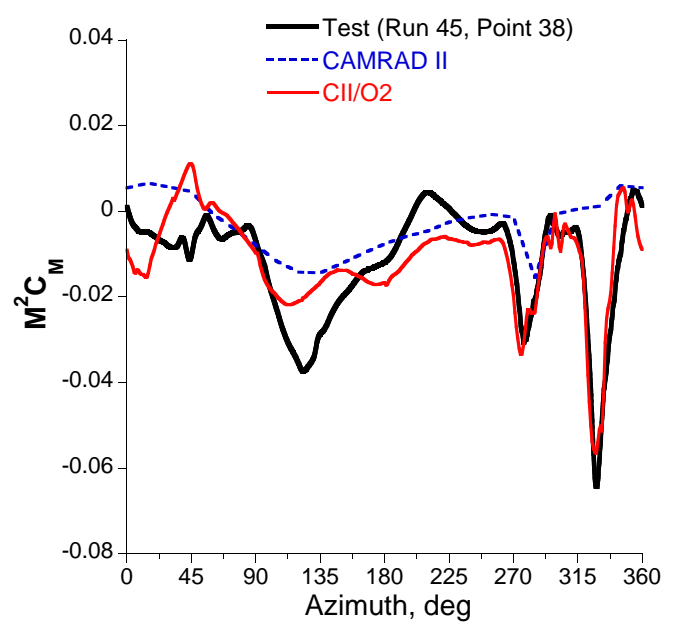

(b) $\mu=0.3, C_{T} / \sigma=0.1255$

Fig. 17: Calculated and measured blade section pitching moment at $\mathrm{r} / \mathrm{R}=0.92$ for thrust sweep.

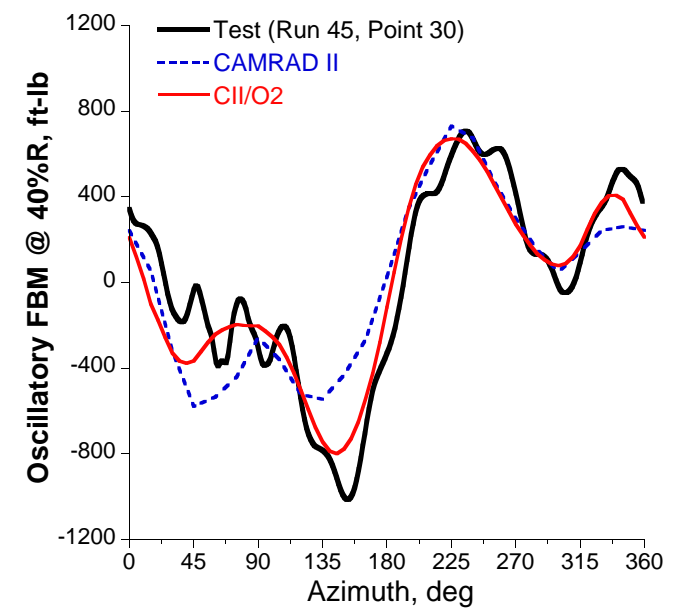

(a) $\mu=0.3, C_{T} / \sigma=0.06$

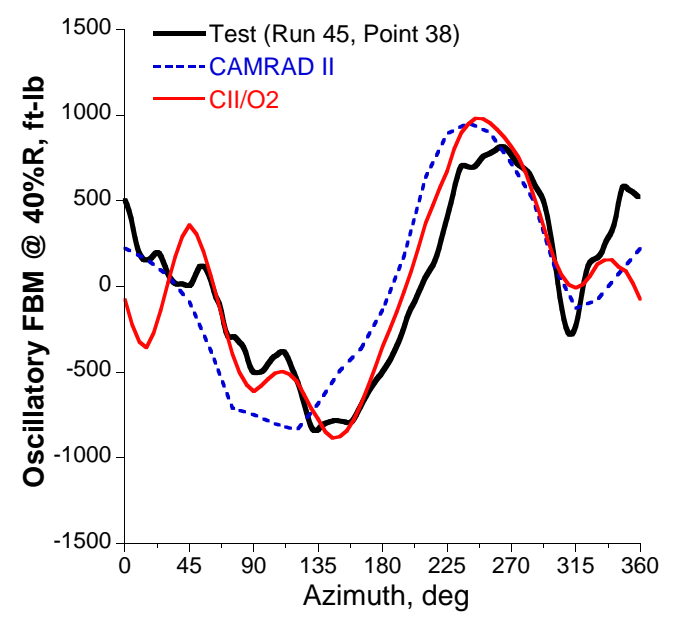

(b) $\mu=0.3, C_{T} / \sigma=0.1255$

Fig. 18: Calculated and measured blade oscillatory flap bending moment at $\mathrm{r} / \mathrm{R}=0.4$ for thrust sweep.

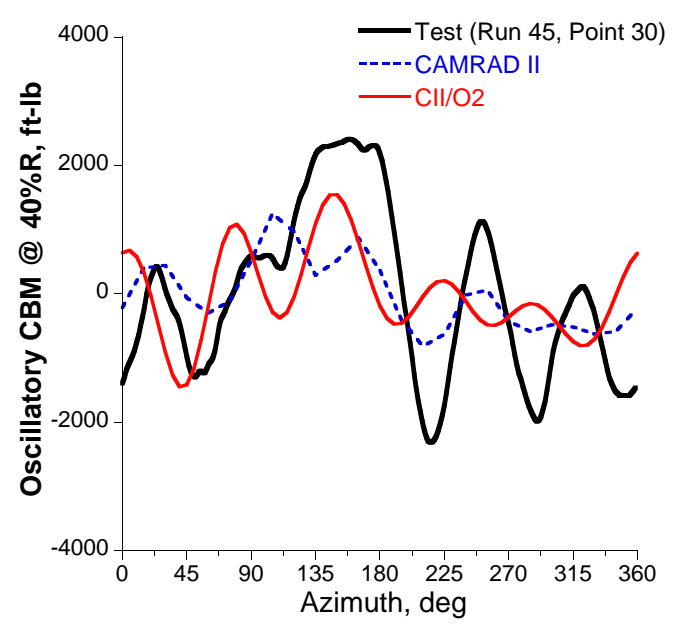

(a) $\mu=0.3, C_{T} / \sigma=0.06$

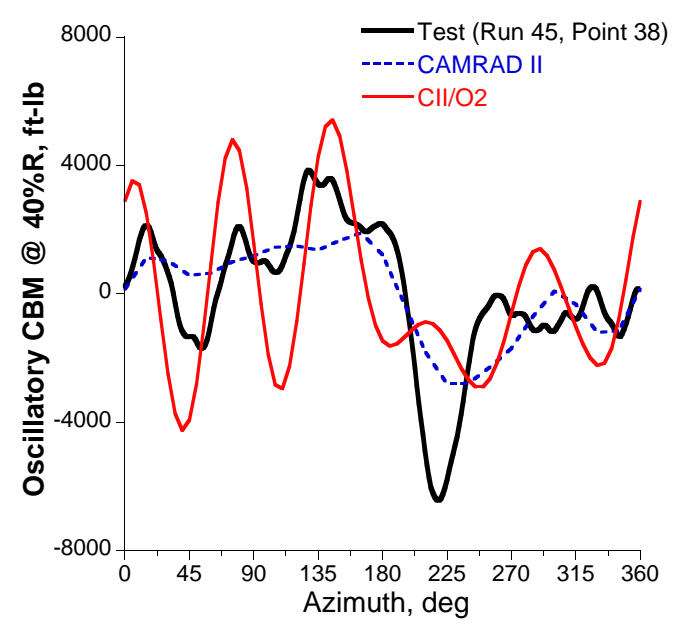

(b) $\mu=0.3, C_{T} / \sigma=0.1255$

Fig. 19: Calculated and measured blade oscillatory chord bending moment at $r / R=0.4$ for thrust sweep. 


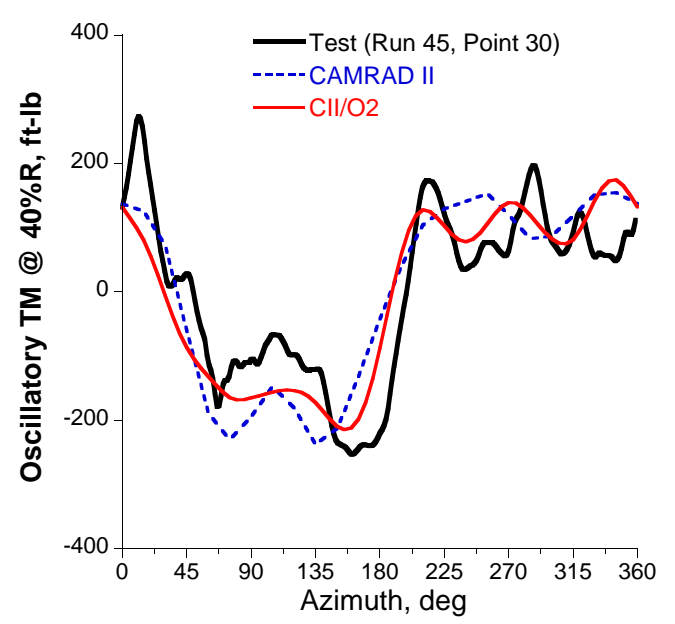

(a) $\mu=0.3, C_{T} / \sigma=0.06$

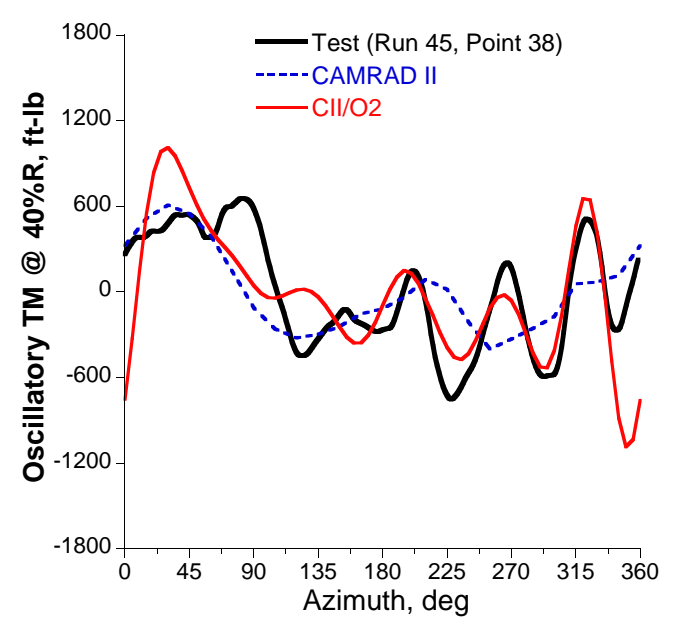

(b) $\mu=0.3, C_{T} / \sigma=0.1255$

Fig. 20: Calculated and measured blade oscillatory torsion moment at $\mathrm{r} / \mathrm{R}=0.4$ for thrust sweep.

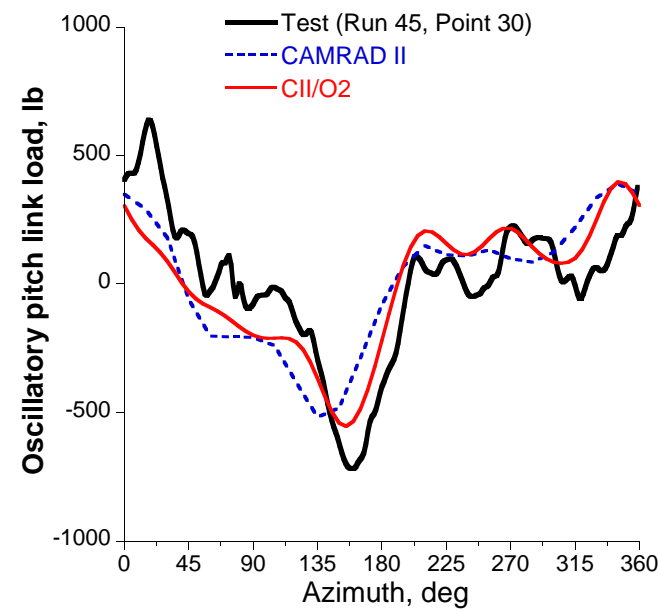

(a) $\mu=0.3, C_{T} / \sigma=0.06$

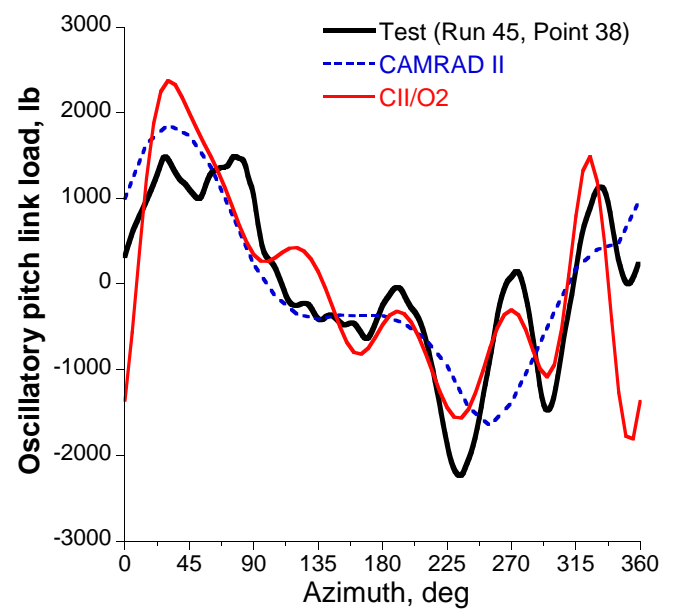

(b) $\mu=0.3, C_{T} / \sigma=0.1255$

Fig. 21: Calculated and measured oscillatory pitch link load for thrust sweep.

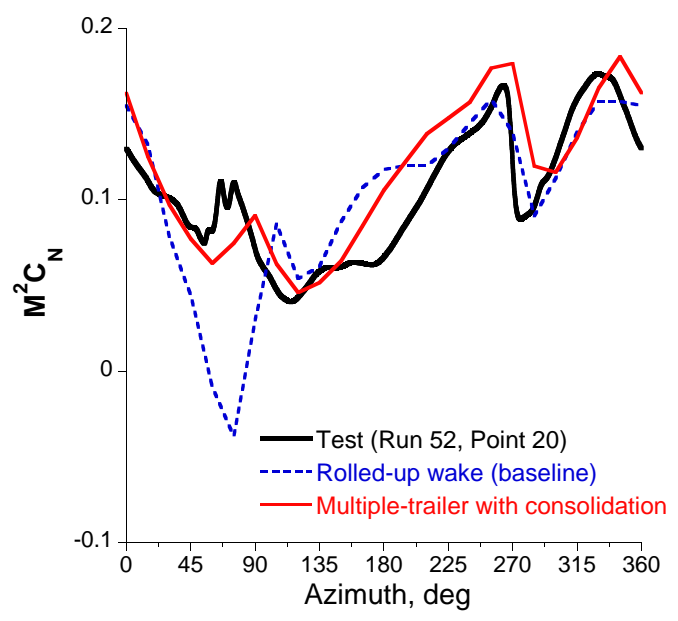

(a) Blade section normal force at $\mathrm{r} / \mathrm{R}=0.92$

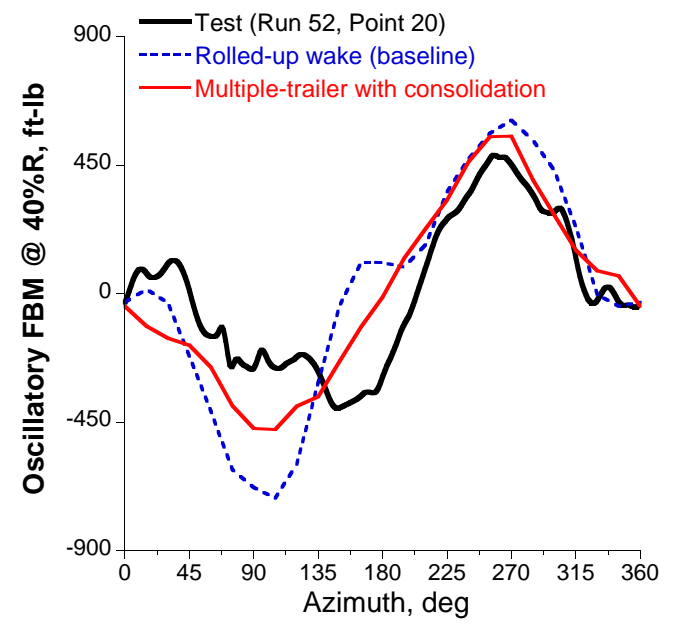

(b) Flap bending moment at $\mathrm{r} / \mathrm{R}=0.4$

Fig. 22: Effects of wake modeling, $\mu=0.2, C_{L} / \sigma=0.92$. 


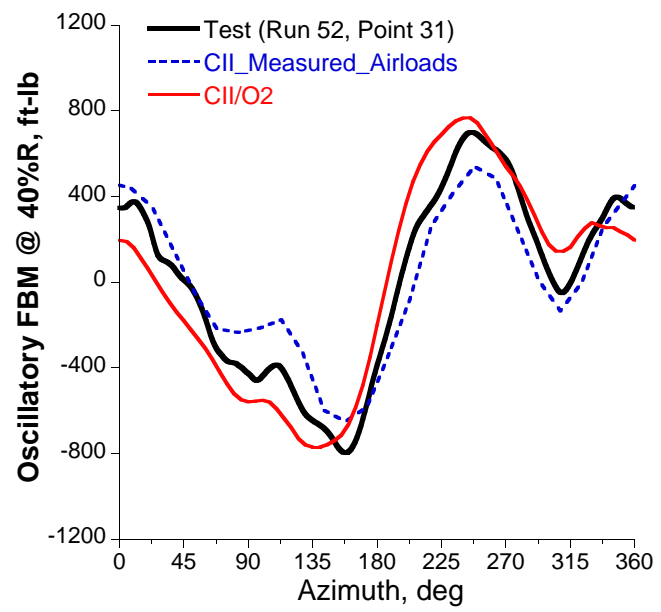

(a) Flap bending moment

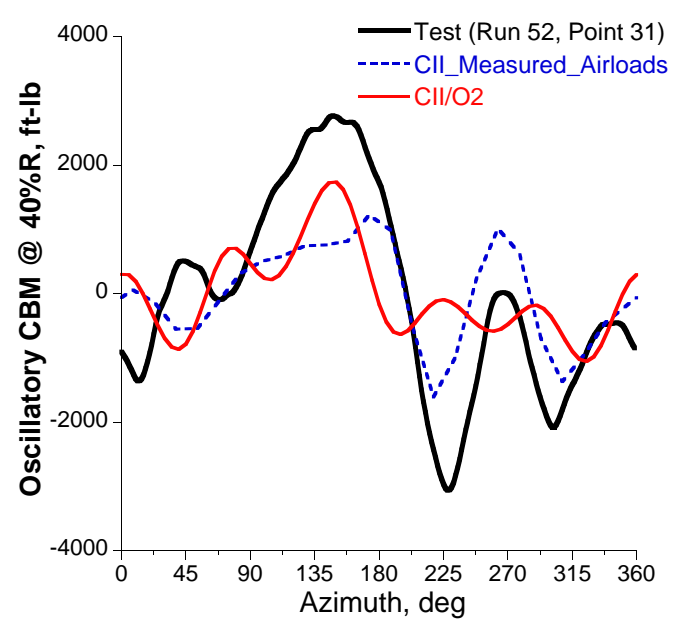

(b) Chord bending moment

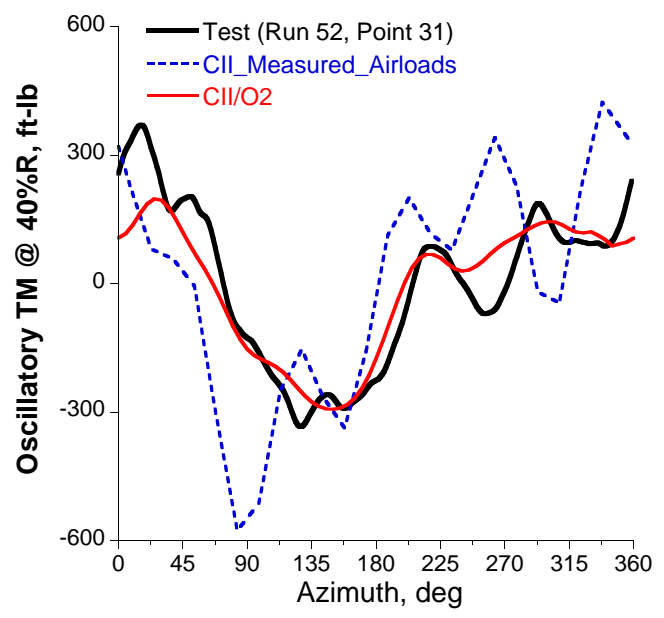

(c) Torsion moment

Fig. 23: Structural loads at $40 \% \mathrm{R}$ calculated with prescribed measured airloads, $\mu=0.3, C_{L} / \sigma=0.09$

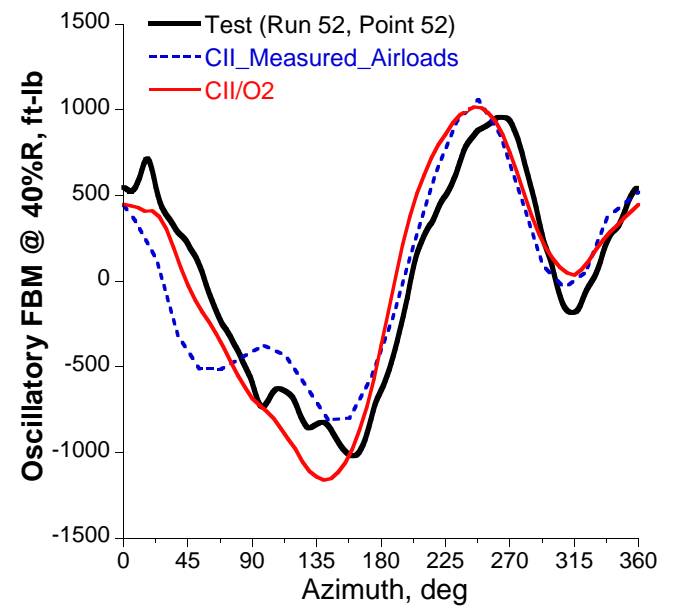

(a) Flap bending moment

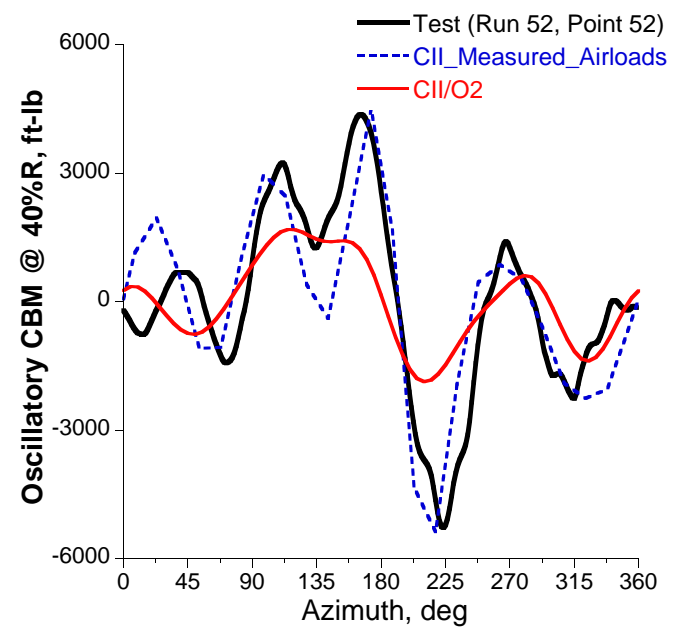

(b) Chord bending moment

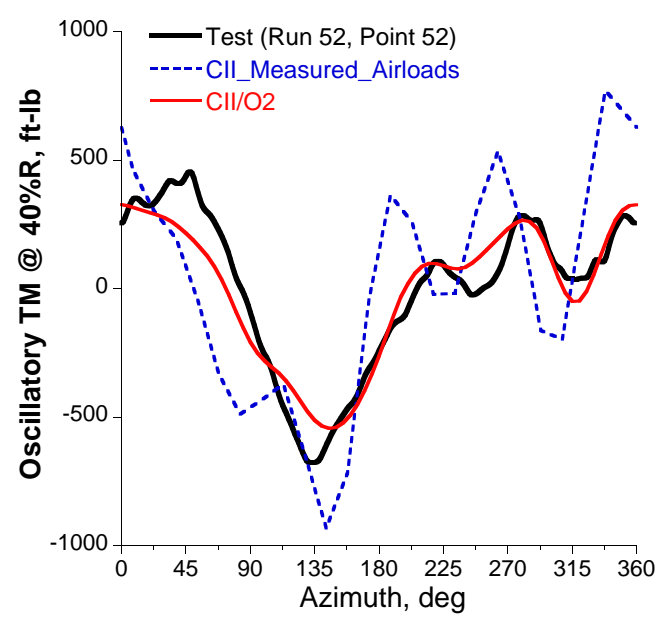

(c) Torsion moment
Fig. 24: Structural loads at $40 \% \mathrm{R}$ calculated with prescribed measured airloads, $\mu=0.4, C_{L} / \sigma=0.09$ 


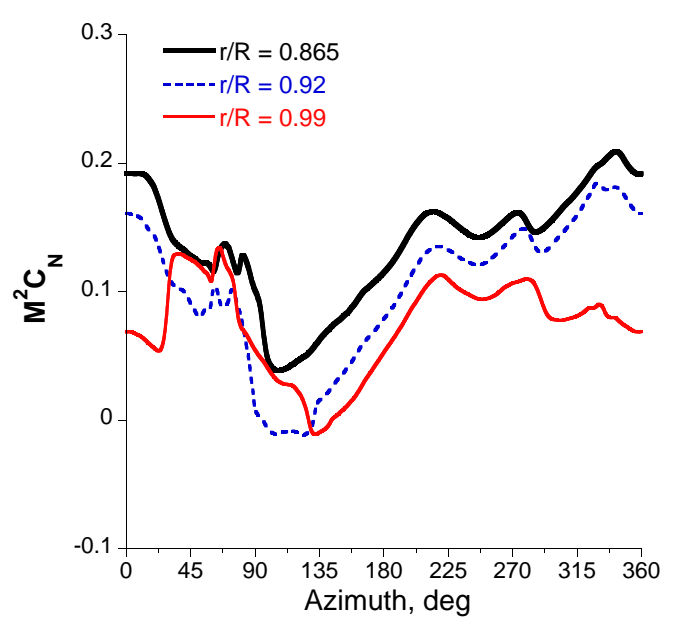

(a) Blade section normal force

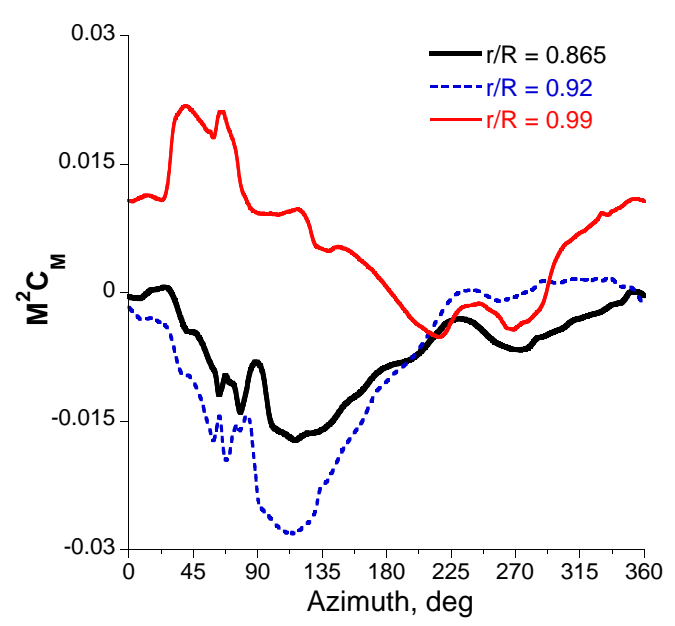

(b) Blade section pitching moment

Fig. 25: Measured blade section airloads, $\mu=0.3, C_{L} / \sigma=0.09$

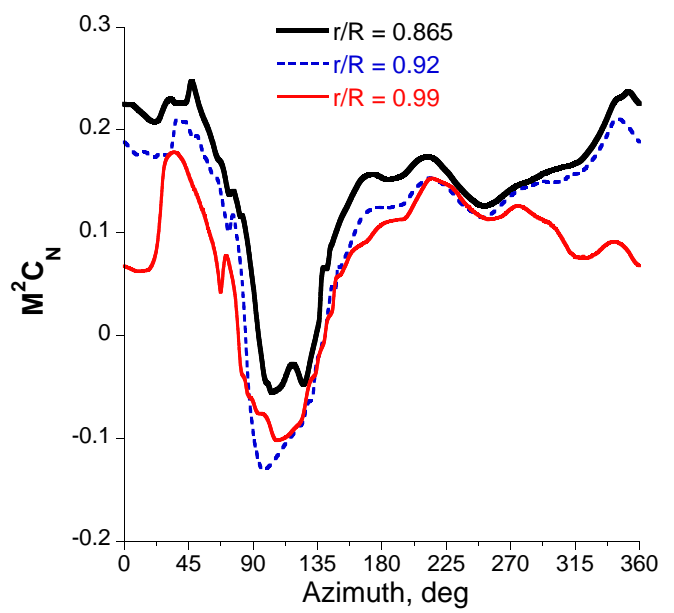

(a) Blade section normal force

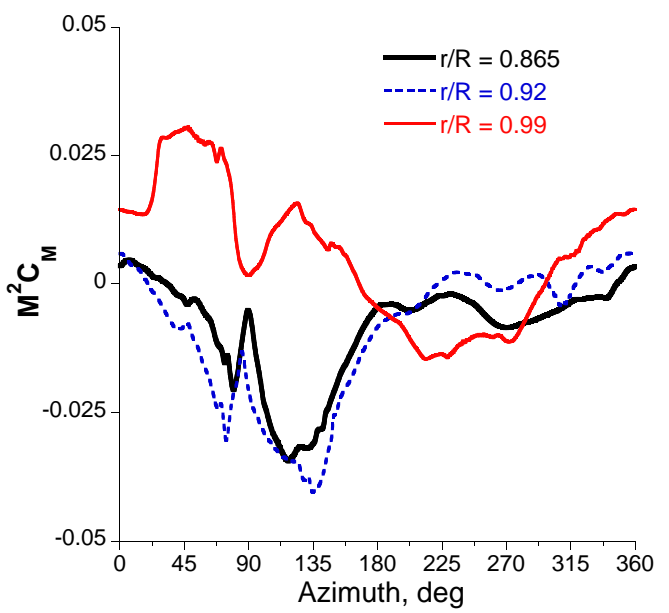

(b) Blade section pitching moment

Fig. 26: Measured blade section airloads, $\mu=0.4, C_{L} / \sigma=0.09$

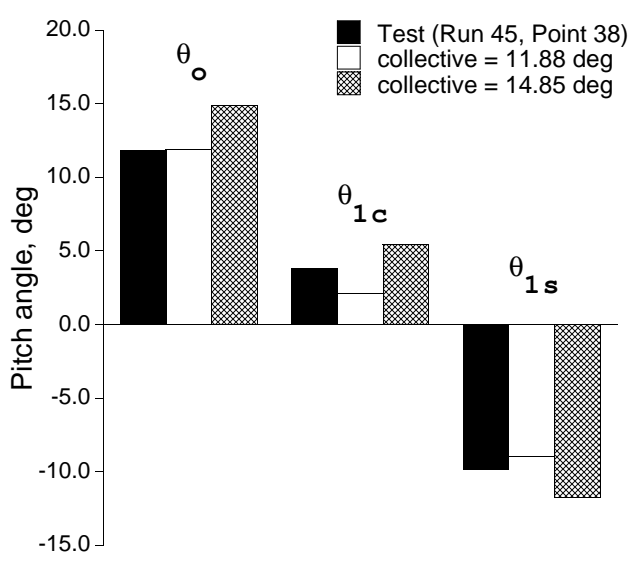

(a) Pitch angle with collective variation

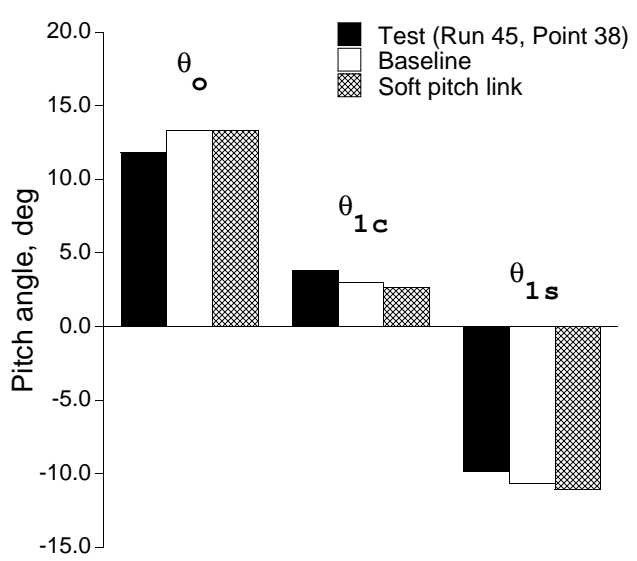

(b) Pitch angle with pitch link stiffness variation

Fig. 27: Pitch angle and thrust, $\mu=0.3, C_{T} / \sigma=0.1255$. 


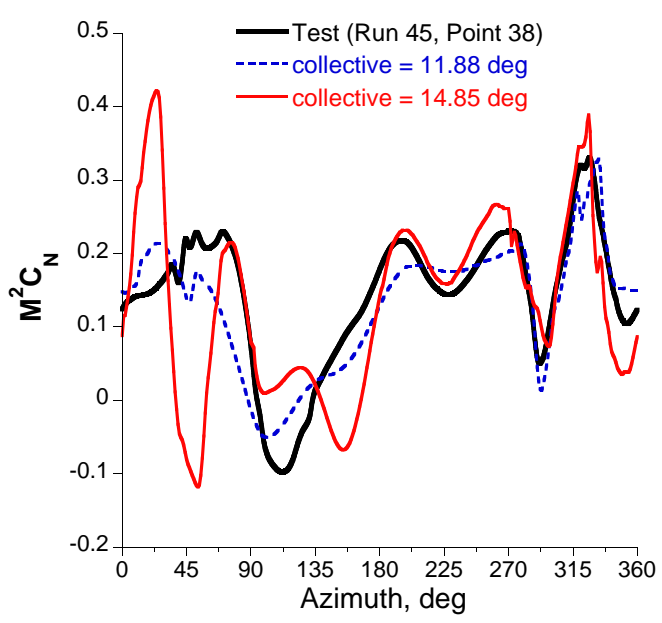

(a) Section normal force with collective variation

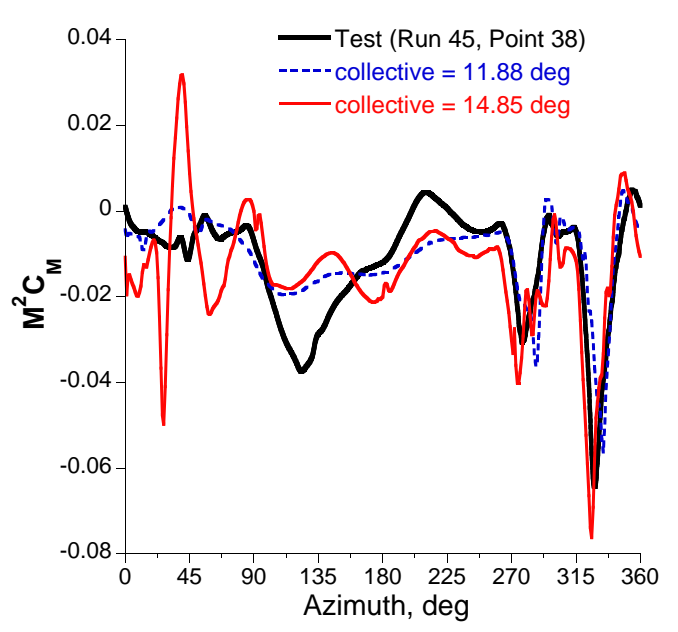

(c) Section pitching moment with collective variation

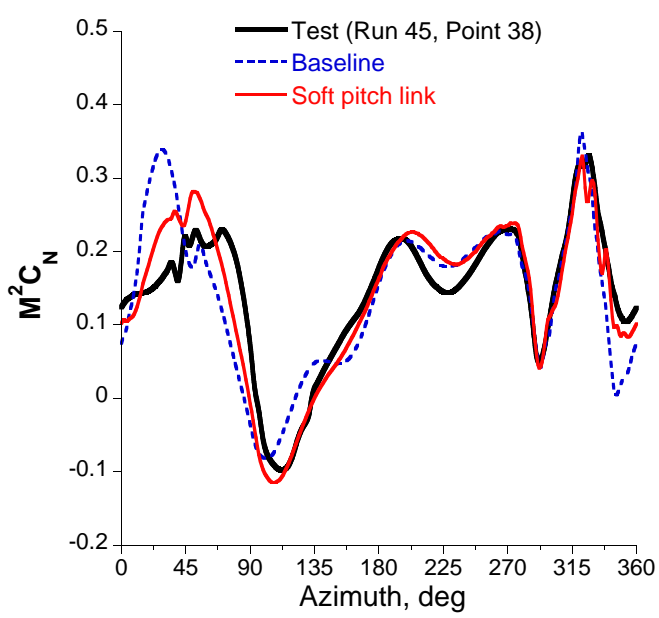

(b) Section normal force with pitch link stiffness variation

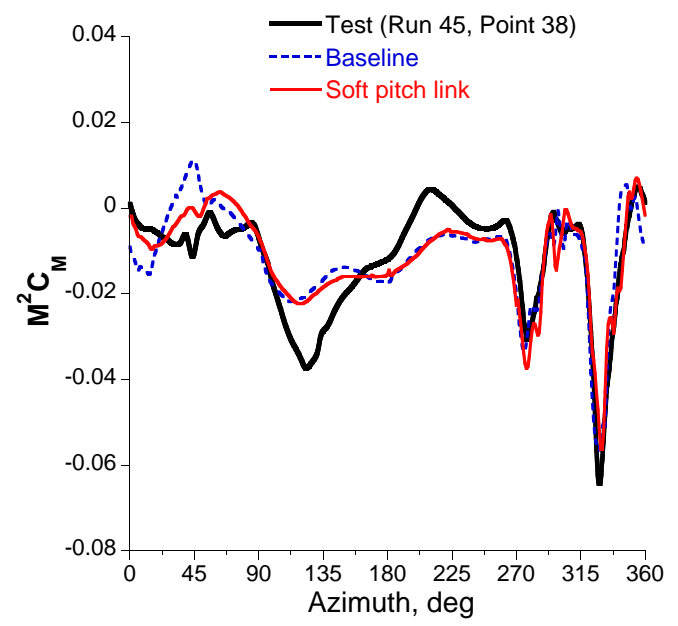

(d) Section pitching moment with pitch link stiffness variation

Fig. 28: Blade section airloads at $\mathrm{r} / \mathrm{R}=0.92, \mu=0.3, C_{T} / \sigma=0.1255$. 


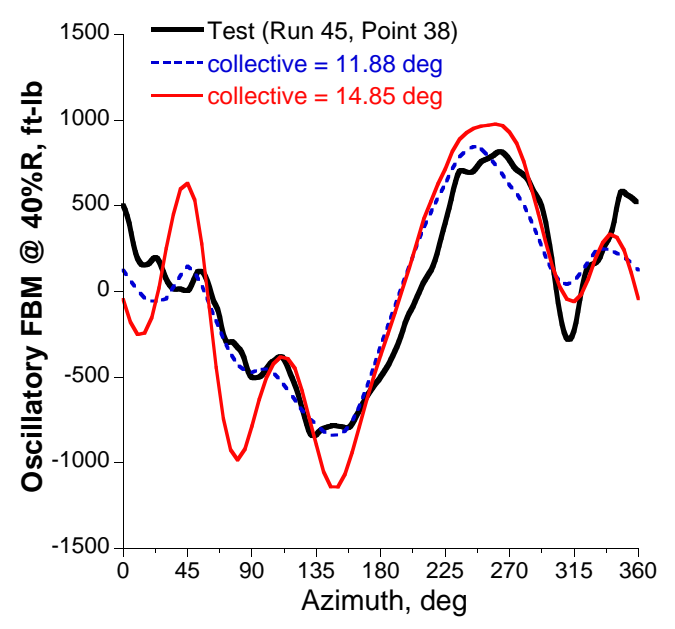

(a) Flap bending moment at $\mathrm{r} / \mathrm{R}=0.4$

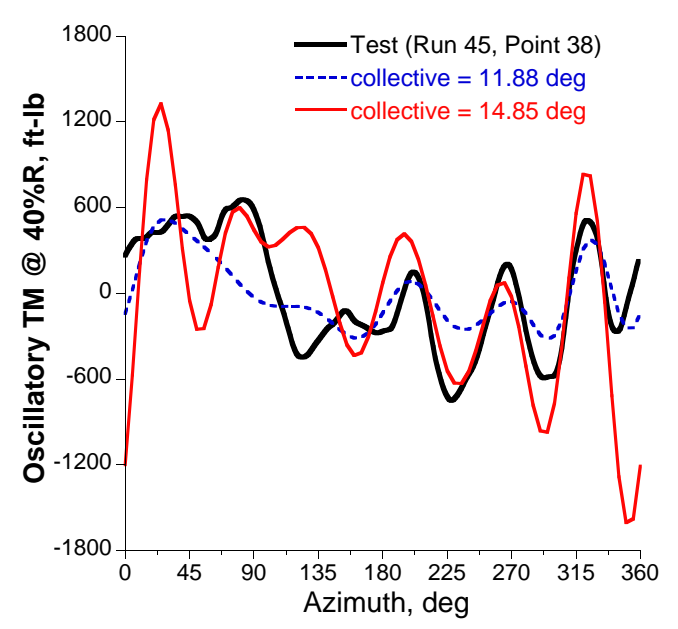

(c) Torsion moment at $\mathrm{r} / \mathrm{R}=0.4$

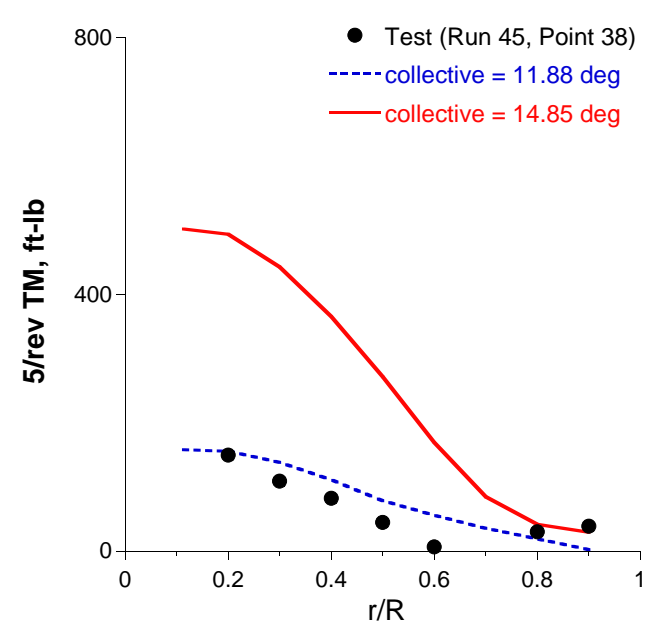

(e) $5 /$ rev torsion moment

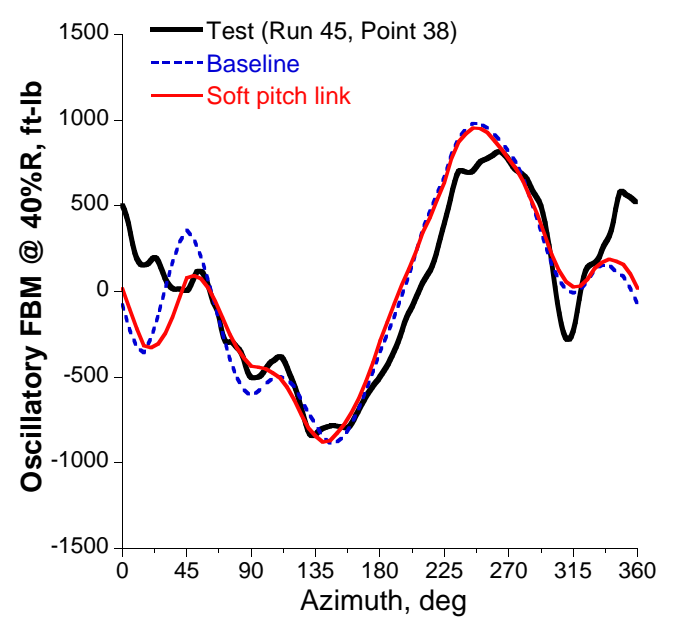

(b) Flap bending moment at $\mathrm{r} / \mathrm{R}=0.4$

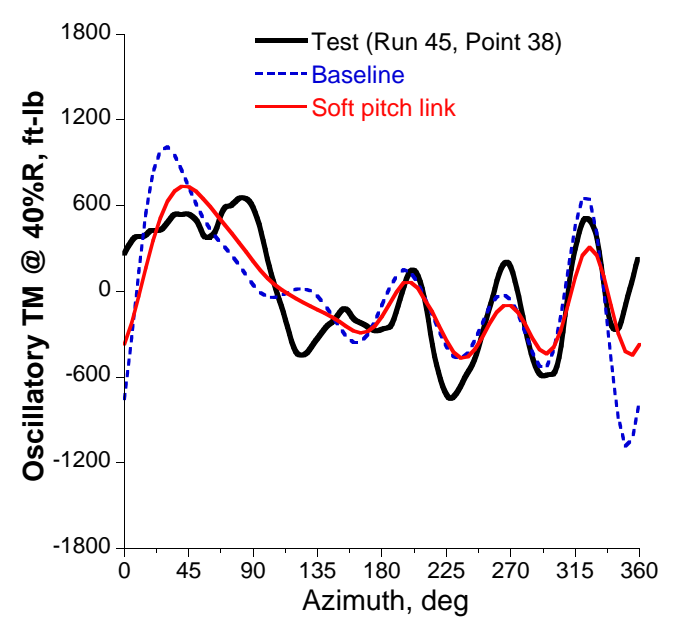

(d) Torsion moment at $\mathrm{r} / \mathrm{R}=0.4$

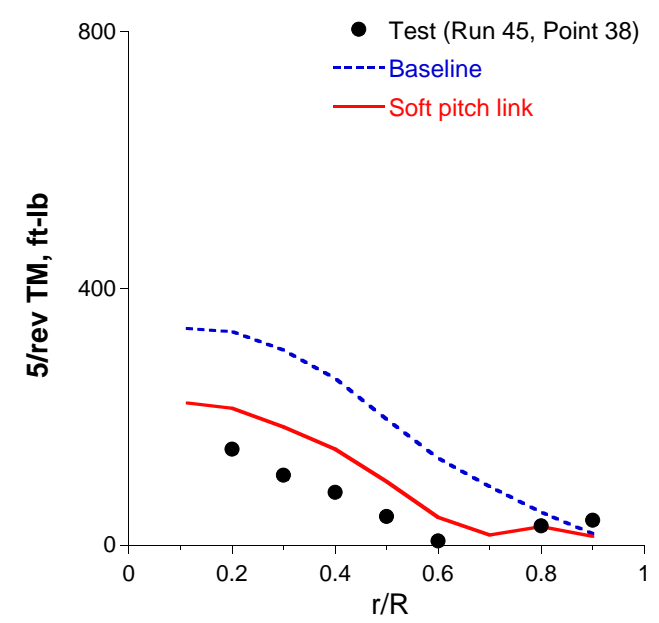

(f) $5 /$ rev torsion moment

Fig. 29: Oscillatory structural loads, $\mu=0.3, C_{T} / \sigma=0.1255$. 